UDC 517.9

N. A. Kachanovsky (Inst. Math. Nat. Acad. Sci. Ukraine, Kyiv)

\title{
GENERALIZED STOCHASTIC DERIVATIVES ON SPACES OF NONREGULAR GENERALIZED FUNCTIONS OF MEIXNER WHITE NOISE
}

\section{УЗАГАЛЬНЕНІ СТОХАСТИЧНІ ПОХІДНІ НА ПОВ'ЯЗАНИХ ІЗ БІЛИМ ШУМОМ МАЙКСНЕРА ПРОСТОРАХ НЕРЕГУЛЯРНИХ УЗАГАЛЬНЕНИХ ФУНКЦІЙ}

We introduce and study generalized stochastic derivatives on the Kondratiev-type spaces of nonregular generalized functions of Meixner white noise. Properties of these derivatives are quite analogous to the properties of the stochastic derivatives in the Gaussian analysis. As an example we calculate the generalized stochastic derivative of the solution of some stochastic equation with a Wick-type nonlinearity.

Вводяться та вивчаються узагальнені стохастичні похідні на пов'язаних із білим шумом Майкснера просторах типу Кондратьєва нерегулярних узагальнених функцій. Властивості цих похідних аналогічні властивостям стохастичних похідних у гауссівському аналізі. Як приклад обчислено узагальнену стохастичну похідну розв' язку певного стохастичного рівняння 3 нелінійністю типу Віка.

Introduction. Let $S^{\prime}$ be the Schwartz distributions space, $\mu$ be the Gaussian measure on $S^{\prime}$. As is well known, every square integrable function $f \in L^{2}\left(S^{\prime}, \mu\right)$ can be presented in the form

$$
f=\sum_{n=0}^{\infty}\left\langle H_{n}, f^{(n)}\right\rangle
$$

where $\left\{\left\langle H_{n}, f^{(n)}\right\rangle\right\}_{n=0}^{\infty}$ are the generalized Hermite polynomials, $f^{(n)} \in \mathcal{H}^{\hat{\otimes} n}, \mathcal{H}$ (in the simplest case) is the complexification of $L^{2}(\mathbb{R}), \hat{\otimes}$ denotes a symmetric tensor product. A stochastic derivative $\mathcal{D}: L^{2}\left(\mathcal{S}^{\prime}, \mu\right) \rightarrow \mathcal{L}\left(\mathcal{H}, L^{2}\left(\mathcal{S}^{\prime}, \mu\right)\right)$ can be defined on its domain

$$
\left\{f \in L^{2}\left(\mathcal{S}^{\prime}, \mu\right): \sum_{n=1}^{\infty} n ! n\left|f^{(n)}\right|_{\mathcal{H}^{\otimes n}}^{2}<\infty\right\}
$$

by the formula

$$
(\mathcal{D} f)\left(g^{(1)}\right):=\sum_{n=1}^{\infty} n\left\langle H_{n-1},\left\langle f^{(n)}, g^{(1)}\right\rangle\right\rangle \quad \forall g^{(1)} \in \mathcal{H},
$$

where $\left\langle f^{(n)}, g^{(1)}\right\rangle \in \mathcal{H}^{\hat{\otimes} n-1}$ is defined by

$$
\left\langle\left\langle f^{(n)}, g^{(1)}\right\rangle, h^{(n-1)}\right\rangle:=\left\langle f^{(n)}, g^{(1)} \hat{\otimes} h^{(n-1)}\right\rangle \quad \forall h^{(n-1)} \in \mathcal{H}^{\hat{\otimes} n-1}
$$

(here $\langle\cdot, \cdot\rangle$ denotes the scalar product in $\mathcal{H}^{\hat{\otimes} n}$ ).

In the paper [1] Fred E. Benth extended the derivative $\mathcal{D}$ on the Kondratiev space of nonregular generalized functions $\left(S^{\prime}\right)^{-1}$ (elements of $\left(S^{\prime}\right)^{-1}$ can be presented in the similar to (0.1) form, but the kernels $\left\{f^{(n)}\right\}_{n=0}^{\infty}$ are singular). This generalization is useful for different applications. For example, as distinct from $L^{2}\left(S^{\prime}, \mu\right)$ in the space $(S)^{-1}$ one can introduce the Wick product $\diamond$ by setting for the Hermite polynomials 


$$
\left\langle H_{n}, f^{(n)}\right\rangle \diamond\left\langle H_{m}, g^{(m)}\right\rangle:=\left\langle H_{n+m}, f^{(n)} \hat{\otimes} g^{(m)}\right\rangle,
$$

and $\mathcal{D}$ is a differentiation with respect to $\diamond$ : for all $F, G \in(S)^{-1} \mathcal{D}(F \diamond G)=$ $=(\mathcal{D} F) \diamond G+F \diamond(\mathcal{D} G)$. Using this result (and another properties of $\mathcal{D}$ ) one can study properties of solutions of stochastic equations with Wick type nonlinearity. Another possible applications are connected with the fact that the stochastic derivative is the adjoint operator to the extended (Skorokhod) stochastic integral.

In the papers $[2,3]$ the author generalized the results of [1] to the spaces of generalized functions of the so-called Gamma white noise analysis (i.e., instead of the Gaussian measure the introduced in [4] Gamma measure $\gamma$ on $\mathcal{S}^{\prime}$ was used). Since the Gamma measure has no the so-called Chaotic Representation Property (i.e., a function $f \in L^{2}\left(S^{\prime}, \gamma\right)$ can not be presented in complete analogy with (0.1), generally speaking) and has some another peculiarities, the corresponding spaces have a more complicated than in the Gaussian analysis structure: nevertheless a natural and rich in content ana$\log$ of the Gaussian theory is possible.

A next natural step consists in the construction of a theory of stochastic differentiation on the generalized functions spaces of the so-called Meixner analysis. In fact, the (introduced in [5]) generalized Meixner measure $\mu$ on the Schwartz distributions space $D^{\prime}$ (the base measure of the Meixner analysis) is a direct generalization of "classical" measures on $D^{\prime}$, such as the Gaussian, Poisson and Gamma measures. This measure is very general, but still has some "classical" properties (for example, the orthogonal polynomials in $L^{2}\left(D^{\prime}, \mu\right)$ are Schefer (generalized Appell in another terminology) ones), therefore a consrtuctive theory is still possible.

In this paper we construct and study generalized stochastic derivatives on the Kondratiev-type (finite order) spaces of nonregular generalized functions of Meixner white noise. These spaces (cf. [6]) are less wide (and therefore more convenient for applications) than the "classical" Kondratiev spaces of generalized functions (e.g., [7 10]), but have all necessary for our considerations properties of Kondratiev spaces (in particular, one can consider the Wick calculus and stochastic equations with a Wick type nonlinearity). Generalized stochastic derivatives on the Kondratiev-type space of regular generalized functions will be considered in the forthcoming paper.

The paper is organized in the following manner. In the first section we give a necessary information about the generalized Meixner measure, spaces of test and generalized functions, the stochastic integration and the Wick calculus. The second section is devoted to generalized stochastic derivatives on the spaces of nonregular generalized functions.

1. Preliminaries. Let $\sigma$ be a measure on $\left(\mathbb{R}_{+}, \mathcal{B}\left(\mathbb{R}_{+}\right)\right.$) (here $\mathcal{B}$ denotes the Borel $\sigma$-algebra) satisfying the following assumptions:

1) $\sigma$ is absolutely continuous with respect to the Lebesgue measure and the density is an infinite differentiable function on $\mathbb{R}_{+}$;

2) $\sigma$ is a nondegenerate measure, i.e., for each nonempty open set $O \subset \mathbb{R}_{+}$ $\sigma(O)>0$.

Remark 1.1. Note that these assumptions are the "simplest sufficient ones" for our considerations; actually it is possible to consider a much more general $\sigma$.

By $D$ denote the set of all real-valued infinite differentiable functions on $\mathbb{R}_{+}$with compact supports. This set can be naturally endowed with a (projective limit) topology of a nuclear space (by analogy with, e.g., [11]): $D=\operatorname{pr} \lim _{\tau \in T} \mathcal{H}_{\tau}$, where $T$ is the set of all pairs $\tau=\left(\tau_{1}, \tau_{2}\right), \tau_{1} \in \mathbb{N}, \tau_{2}$ is an infinite differentiable function on $\mathbb{R}_{+}$ 
such that $\tau_{2}(t) \geq 1 \quad \forall t \in \mathbb{R}_{+} ; \mathcal{H}_{\tau}=\mathcal{H}_{\left(\tau_{1}, \tau_{2}\right)}$ is the Sobolev space on $\mathbb{R}_{+}$of order $\tau_{1}$ weighted by the function $\tau_{2}$, i.e., the scalar product in $\mathcal{H}_{\tau}$ is given by the formula

$$
(f, g)_{\tau}:=(f, g)_{\mathcal{H}_{\tau}}=\int_{\mathbb{R}_{+}}\left(f(t) g(t)+\sum_{k=1}^{\tau_{1}} f^{(k)}(t) g^{(k)}(t)\right) \tau_{2}(t) \sigma(d t) .
$$

Hence in what follows, we understand $D$ as the corresponding topological space.

Let $\mathcal{H}_{\tau, \mathbb{C}}:=\mathcal{H}_{\tau} \oplus i \mathcal{H}_{\tau}$ be the complexification of $\mathcal{H}_{\tau}$ (here and below by the subindex $\mathbb{C}$ denote complexifications of spaces). By $|\cdot|_{\tau}$ denote the corresponding to the scalar product $(\cdot, \cdot)_{\tau}$ norm in $\mathcal{H}_{\tau, \mathbb{C}}$, i.e., $|f|_{\tau}^{2}=(f, \bar{f})_{\tau}$.

Let us consider the (nuclear) chain (the rigging of $L^{2}\left(\mathbb{R}_{+}, \sigma\right)$ - the space of square integrable with respect to $\sigma$ real-valued functions on $\mathbb{R}_{+}$)

$$
\begin{gathered}
D^{\prime}=\underset{\tau^{\prime} \in T}{\operatorname{ind}} \lim \mathcal{H}_{-\tau^{\prime}} \supset \mathcal{H}_{-\tau} \supset L^{2}\left(\mathbb{R}_{+}, \sigma\right)=: \mathcal{H} \supset \\
\supset \mathcal{H}_{\tau} \supset \operatorname{pr} \lim _{\tau^{\prime} \in T} \mathcal{H}_{\tau^{\prime}}=D,
\end{gathered}
$$

where $\mathcal{H}_{-\tau}, D^{\prime}$ are the dual to $\mathcal{H}_{\tau}, D$ with respect to $\mathcal{H}$ spaces correspondingly. By $|\cdot|_{-\tau}$ and $|\cdot|_{0}$ denote the norms in $\mathcal{H}_{-\tau}$ and $\mathcal{H}$. Let $\langle\cdot, \cdot\rangle$ be the generated by the scalar product in $\mathcal{H}$ dual pairing between elements of $D^{\prime}$ and $D$ (and also $\mathcal{H}_{-\tau}$ and $\left.\mathcal{H}_{\tau}\right)$. The notation $|\cdot|_{\tau},|\cdot|_{0},|\cdot|_{-\tau},(\cdot, \cdot)_{\tau}$, and $\langle\cdot, \cdot\rangle$ will be preserved for tensor powers and complexifications of spaces.

Remark 1.2. Note that all scalar products and pairings in this paper are real, i.e., they are bilinear functionals. In particular, $\langle\cdot, \cdot\rangle$ is a real pairing in complexifications of spaces.

Let us fix arbitrary functions $\alpha, \beta: \mathbb{R}_{+} \rightarrow \mathbb{C}$ that are smooth and satisfy

$$
\theta:=-\alpha-\beta \in \mathbb{R}, \quad \eta:=\alpha \beta \in \mathbb{R}_{+},
$$

$\theta$ and $\eta$ are bounded on $\mathbb{R}_{+}$(note that in a sense $\eta$ is a "key parameter" and will be mentioned very often below). Further, let $\tilde{v}(\alpha, \beta, d s)$ be a probability measure on $\mathbb{R}$ that is defined by its Fourier transform

$$
\begin{gathered}
\int_{\mathbb{R}} e^{i u s} \tilde{v}(\alpha, \beta, d s)= \\
=\exp \left\{-i u(\alpha+\beta)+2 \alpha \beta \sum_{m=1}^{\infty} \frac{(\alpha \beta)^{m-1}}{m}\left[\sum_{n=2}^{\infty} \frac{(-i u)^{n}}{n !}\left(\beta^{n-2}+\beta^{n-3} \alpha+\ldots+\alpha^{n-2}\right)\right]^{m}\right\}, \\
v(\alpha, \beta, d s):=\frac{1}{s^{2}} \tilde{v}(\alpha, \beta, d s) .
\end{gathered}
$$

Definition 1.1. We say that the probability measure $\mu$ on the measurable space $\left(D^{\prime}, \mathcal{F}\right)$ (here $\mathcal{F}$ is the generated by cylinder sets $\sigma$-algebra on $\left.D^{\prime}\right)$ with the Fourier transform

$$
\int_{D^{\prime}} e^{i\langle x, \xi\rangle} \mu(d x)=\exp \left\{\int_{\mathbb{R}_{+}} \sigma(d t) \int_{\mathbb{R}} v(\alpha(t), \beta(t), d s)\left(e^{i s \xi(t)}-1-i s \xi(t)\right)\right\}
$$

(here $\xi \in D$ ) is called the generalized Meixner measure.

Theorem 1.1 [5]. The generalized Meixner measure $\mu$ is a generalized sto- 
chastic process with independent values in the sense of [12]. The Laplace transform of $\mu$ is given in a neighborhood of zero $\mathcal{U}_{0} \subset D_{\mathbb{C}}$ by the following formula:

$$
\begin{gathered}
l_{\mu}(\lambda)=\int_{D^{\prime}} e^{\langle x, \lambda\rangle} \mu(d x)=\exp \left\{\int_{\mathbb{R}_{+}} \sum_{m=1}^{\infty} \frac{(\alpha(t) \beta(t))^{m-1}}{m} \times\right. \\
\left.\times\left(\sum_{m=1}^{\infty} \frac{(-\lambda)^{n}}{n !}\left(\beta(t)^{n-2}+\beta(t)^{n-3} \alpha(t)+\ldots+\alpha(t)^{n-2}\right)\right)^{m} \sigma(d t)\right\}, \quad \lambda \in \mathcal{U}_{0} .
\end{gathered}
$$

Remark 1.3. Accordingly to the classical classification [13] (see also [14, 5]) for $\alpha=\beta=0$ (here and below all such equalities we understand $\sigma$-a.e.) $\mu$ is the Gaussian measure: for $\alpha \neq 0$ (here and below $a(\cdot) \neq b(\cdot)$ means that $a-b \neq 0$ on some measurable set $M$ such that $\sigma(M)>0$ ), $\beta=0 \mu$ is the centered Poissonian measure; for $\alpha=\beta \neq 0 \mu$ is the centered Gamma measure; for $\alpha \neq \beta, \alpha \beta \neq 0$, $\alpha, \beta: \mathbb{R}_{+} \rightarrow \mathbb{R} \mu$ is the centered Pascal measure; for $\alpha=\bar{\beta}, \operatorname{Im}(\alpha) \neq 0 \mu$ is the centered Meixner measure.

The following statement describes an important property of $\mu$.

Lemma 1.1 [15]. There exists $\tilde{\tau} \in T$ such that the generalized Meixner measure is concentrated on $\mathcal{H}_{-\tilde{\tau}}$, i.e., $\mu\left(\mathcal{H}_{-\tilde{\tau}}\right)=1$.

Remark 1.4. In what follows, we assume that $\mu$ is concentrated on $\mathcal{H}_{-\tau}$ for all $\tau \in T$. In fact, it is sufficient to exclude from $T$ the indexes $\tau$ such that $\mu$ is not concentrated on $\mathcal{H}_{-\tau}$.

Now by $\left(L^{2}\right)=L^{2}\left(D^{\prime}, \mu\right)$ denote the space of square integrable with respect to $\mu$ complex-valued functions on $D^{\prime}$. Let us construct orthogonal polynomials on $\left(L^{2}\right)$.

Definition 1.2. We define a so-called Wick exponential (a generating function of the orthogonal polynomials) by setting

$$
\begin{aligned}
& : \exp (x ; \lambda): \\
& \stackrel{\mathrm{df}}{=} \exp \left\{-\int_{\mathbb{R}_{+}}\left(\frac{\lambda(t)^{2}}{2}+\sum_{n=3}^{\infty} \frac{\lambda(t)^{n}}{n}\left(\alpha(t)^{n-2}+\alpha(t)^{n-3} \beta(t)+\ldots+\beta(t)^{n-2}\right)\right) \sigma(d t)+\right. \\
& \left.+\left\langle x, \lambda+\sum_{n=2}^{\infty} \frac{\lambda^{n}}{n}\left(\alpha^{n-1}+\alpha^{n-2} \beta+\ldots+\beta^{n-1}\right)\right\rangle\right\},
\end{aligned}
$$

where $\lambda \in \mathcal{U}_{0} \subset D_{\mathbb{C}}, x \in D^{\prime}, \mathcal{U}_{0}$ is some neighborhood of $0 \in D_{\mathbb{C}}$.

Remark 1.5. It was proved in [5] that

$$
: \exp (x ; \lambda):=\frac{e^{\langle x, \Psi(\lambda)\rangle}}{l_{\mu}(\Psi(\lambda))}
$$

with

$$
\Psi(\lambda)=\lambda+\sum_{n=2}^{\infty} \frac{\lambda^{n}}{n}\left(\alpha^{n-1}+\alpha^{n-2} \beta+\ldots+\beta^{n-1}\right),
$$

therefore $: \exp (x ; \cdot):$ is a generating function of the so-called Schefer polynomials (or the generalized Appell polynomials in another terminology). This fact gives us the possibility to use in our considerations well-known results of the so-called "biorthogonal analysis" (see, e.g., [6-10,16-18] and references therein). 
It is clear (see also [5]) that $: \exp (x ; \cdot):$ is a holomorphic at zero function on $D_{\mathbb{C}}$ for each $x \in D^{\prime}$. Therefore using the Cauchy inequalities (see, e.g., [19]) and the kernel theorem (see, e.g., [11]) one can obtain the representation

$$
: \exp (x ; \lambda):=\sum_{n=0}^{\infty} \frac{1}{n !}\left\langle P_{n}(x), \lambda^{\otimes n}\right\rangle, \quad P_{n}(x) \in D_{\mathbb{C}}^{\prime \otimes} \hat{\otimes}^{\otimes} \quad x \in D^{\prime}, \quad \lambda \in D_{\mathbb{C}} .
$$

Here (and below) $\hat{\otimes}$ denotes a symmetric tensor product, $\lambda^{\otimes 0}=1$ even for $\lambda \equiv 0$.

Remark 1.6. It follows from the given in [5] recurrence formula for $P_{n}(x)$ that actually $P_{n}(x) \in D^{\prime \hat{\otimes}_{n}}$ for $x \in D^{\prime}$. Moreover, if $\tau \in T$ is such that the Dirac deltafunction $\delta_{0} \in \mathcal{H}_{-\tau}$ (it means that $\delta_{s} \in \mathcal{H}_{-\tau} \forall s \in \mathbb{R}_{+}$, see, e.g., [11]) then for $x \in \mathcal{H}_{-\tau}$ we have $P_{n}(x) \in \mathcal{H}_{-\tau}^{\hat{\otimes} n}$.

In what follows, we assume that this statement holds true for all $\tau \in T$. In fact, by analogy with Remark 1.4 it is sufficient to exclude from $T$ the indexes $\tau$ such that $\delta_{0} \notin \mathcal{H}_{-\tau}$.

Definition 1.3. We say that the polynomials $\left\langle P_{n}, f^{(n)}\right\rangle, f^{(n)} \in D_{\mathbb{C}}^{\hat{\otimes}_{n}}, \quad n \in \mathbb{Z}_{+}$, are called the generalized Meixner polynomials.

Remark 1.7. Depending on $\alpha$ and $\beta$ in (1.2) the generalized Meixner polynomials can be the generalized Hermite polynomials $(\alpha=\beta=0)$; the generalized Charlier polynomials $(\alpha \neq 0, \beta=0)$; the generalized Laguerre polynomials $(\alpha=\beta \neq$ $0)$; the Meixner polynomials $\left(\alpha \neq \beta, \alpha \beta \neq 0, \alpha, \beta: \mathbb{R}_{+} \rightarrow \mathbb{R}\right)$; the Meixner Pollaczek polynomials $(\alpha=\bar{\beta}, \operatorname{Im}(\alpha) \neq 0$ ) (see also Remark 1.3).

In order to formulate a statement on an orthogonality of the generalized Meixner polynomials we need the following definition.

Definition 1.4. We define the scalar product $\langle\cdot, \cdot\rangle_{\mathrm{ext}}$ on $D_{\mathbb{C}}^{\hat{\otimes} n}, n \in \mathbb{N}$, by the formula

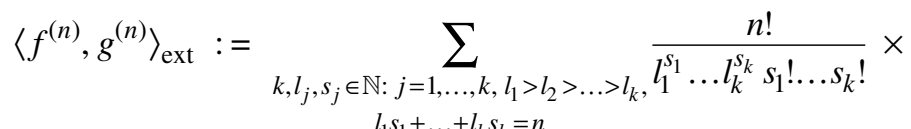

$$
\begin{aligned}
& \times \int_{\mathbb{R}_{+}^{s_{1}+\ldots+s_{k}}} f^{(n)}(\underbrace{t_{1}, \ldots, t_{1}}_{l_{1}}, \ldots, \underbrace{t_{s_{1}}, \ldots, t_{s_{1}}}_{l_{1}}, \ldots, \underbrace{t_{s_{1}+\ldots+s_{k}}, \ldots, t_{s_{1}+\ldots+s_{k}}}_{l_{k}}) \times \\
& \times g^{(n)}(\underbrace{t_{1}, \ldots, t_{1}}_{l_{1}}, \ldots, \underbrace{t_{s_{1}}, \ldots, t_{s_{1}}}_{l_{1}}, \ldots, \underbrace{t_{s_{1}+\ldots+s_{k}}, \ldots, t_{s_{1}}+\ldots+s_{k}}_{l_{k}}) \eta\left(t_{1}\right)^{l_{1}-1} \ldots \eta\left(t_{s_{1}}\right)^{l_{1}-1} \times \\
& \times \eta\left(t_{s_{1}+1}\right)^{l_{2}-1} \ldots \eta\left(t_{s_{1}+s_{2}}\right)^{l_{2}-1} \ldots \eta\left(t_{s_{1}+\ldots+s_{k-1}+1}\right)^{l_{k}-1} \ldots \eta\left(t_{s_{1}+\ldots+s_{k}}\right)^{l_{k}-1} \times \\
& \times \sigma\left(d t_{1}\right) \ldots \sigma\left(d t_{s_{1}+\ldots+s_{k}}\right) .
\end{aligned}
$$

Denote by $|\cdot|_{\text {ext }}$ the corresponding norm, i.e., $\left|f^{(n)}\right|_{\text {ext }}^{2}=\left\langle f^{(n)}, \overline{f^{(n)}}\right\rangle_{\mathrm{ext}}$. For $n=$ $=0\left\langle f^{(0)}, g^{(0)}\right\rangle_{\mathrm{ext}}:=f^{(0)} g^{(0)} \in \mathbb{C},\left|f^{(0)}\right|_{\mathrm{ext}}=\left|f^{(0)}\right|$.

Example. It is easy to see that for $n=1$

$$
\left\langle f^{(1)}, g^{(1)}\right\rangle_{\mathrm{ext}}=\left\langle f^{(1)}, g^{(1)}\right\rangle=\int_{\mathbb{R}_{+}} f^{(1)}(t) g^{(1)}(t) \sigma(d t) .
$$

Further, for $n=2$ 


$$
\left\langle f^{(2)}, g^{(2)}\right\rangle_{\mathrm{ext}}=\left\langle f^{(2)}, g^{(2)}\right\rangle+\int_{\mathbb{R}_{+}} f^{(2)}(t, t) g^{(2)}(t, t) \eta(t) \sigma(d t) .
$$

If $\eta=0$ (this means that $\mu$ is the Gaussian or Poissonian measure, see Remark 1.3) then $\left\langle f^{(n)}, g^{(n)}\right\rangle_{\text {ext }}=\left\langle f^{(n)}, g^{(n)}\right\rangle$ for all $n \in \mathbb{Z}_{+}$; in general, $\left\langle f^{(n)}, g^{(n)}\right\rangle_{\text {ext }}=$ $=\left\langle f^{(n)}, g^{(n)}\right\rangle+\ldots$.

Theorem 1.2 [5]. The generalized Meixner polynomials are orthogonal in $\left(L^{2}\right)$ in the sense that

$$
\int_{D^{\prime}}\left\langle P_{n}(x), f^{(n)}\right\rangle\left\langle P_{m}(x), g^{(m)}\right\rangle \mu(d x)=\delta_{m n} n !\left\langle f^{(n)}, g^{(n)}\right\rangle_{\mathrm{ext}} .
$$

By $\mathcal{H}_{\mathrm{ext}}^{(n)}, n \in \mathbb{N}$, denote the closure of $D_{\mathbb{C}}^{\hat{\otimes} n}$ with respect to the norm $|\cdot|_{\text {ext }}$, $\mathcal{H}_{\mathrm{ext}}^{(0)}:=\mathbb{C}$. Of course, $\mathcal{H}_{\mathrm{ext}}^{(n)}, n \in \mathbb{Z}_{+}$, are Hilbert spaces; for the scalar products in these spaces it is natural to preserve the notation $\langle\cdot, \cdot\rangle_{\text {ext }}$.

Remark 1.8. It is not difficult to prove by analogy with [20] that the space $\mathcal{H}_{\mathrm{ext}}^{(n)}$ is, generally speaking, the orthogonal sum of $\mathcal{H}_{\mathbb{C}}^{\hat{\otimes} n} \equiv L^{2}\left(\mathbb{R}_{+}, \sigma\right)_{\mathbb{C}}^{\hat{\otimes} n}$ and some another Hilbert spaces (as a "limit case" one can consider $\eta=0$, in this case $\mathcal{H}_{\mathrm{ext}}^{(n)}=$ $=\mathcal{H}_{\mathbb{C}}^{\hat{\otimes} n}$ ). In this sense $\mathcal{H}_{\mathrm{ext}}^{(n)}$ is an extension of $\mathcal{H}_{\mathbb{C}}^{\hat{\otimes} n}$.

One can give another explanation of the fact that $\mathcal{H}_{\mathrm{ext}}^{(n)}$ is a more wide space than $\mathcal{H} \underset{\mathbb{C}}{\hat{\otimes} n}$. Namely, let $F^{(n)} \in \mathcal{H}_{\mathbb{C}}^{\hat{\otimes} n}\left(F^{(n)}\right.$ is an equivalence class in $\left.\mathcal{H}_{\mathbb{C}}^{\hat{\otimes} n}\right)$. We select a representative (a function) $\tilde{F}^{(n)} \in F^{(n)}$ with a "zero diagonal", i.e., $\tilde{F}^{(n)}$ is such that $\tilde{F}^{(n)}\left(t_{1}, \ldots, t_{n}\right)=0$ if $t_{i}=t_{j}$ for $i \neq j$, where $i, j \in\{1, \ldots, n\}$. This function generates the equivalence class $\hat{F}^{(n)}$ in $\mathcal{H}_{\text {ext }}^{(n)}$ that can be identified with $F^{(n)}$ (see [15] for details).

Let us recall the construction of the Kondratiev-type spaces of test and generalized functions (see, e.g., [6 - 10, $16-18,21]$ ).

In the classical Gaussian and Poissonian analysis the Kondratiev-type spaces are "based" on the tensor powers of complexification of chain (1.1):

$$
D_{\mathbb{C}}^{\prime \hat{\otimes} n} \supset \mathcal{H}_{-\tau, \mathbb{C}}^{\hat{\otimes} n} \supset \mathcal{H}_{\mathbb{C}}^{\hat{\otimes} n} \supset \mathcal{H}_{\tau, \mathbb{C}}^{\hat{\otimes} n} \supset D_{\mathbb{C}}^{\hat{\otimes} n}, \quad \tau \in T .
$$

But in the light of orthogonality relation (1.3) now it will be more natural to use $\mathcal{H}_{\text {ext }}^{(n)}$ as "central spaces" (by analogy with the Gamma analysis, see, e.g., [22]). In order to construct such chains we need the following proposition.

Proposition 1.1 [15]. There exists $\tilde{\tau} \in T$ such that for each $n \in \mathbb{N} \quad \mathcal{H} \underset{\tilde{\tau}, \mathbb{C}}{\hat{\otimes} n}$ is densely and continuously embedded in $\mathcal{H}_{\mathrm{ext}}^{(n)}$ and, moreover, for all $f^{(n)} \in \mathcal{H} \underset{\tilde{\tau}, \mathbb{C}}{\hat{\otimes} n}$ the estimate

$$
\left|f^{(n)}\right|_{\text {ext }}^{2} \leq n ! c^{n}\left|f^{(n)}\right|_{\tilde{\tau}}^{2}
$$

with some $c>0$ is valid.

Remark 1.9. Let $\mathcal{H}_{\tau}$ be continuously embedded in $\mathcal{H}_{\tilde{\tau}}(\tau, \tilde{\tau} \in T, \tilde{\tau}$ from Proposition 1.1). Then it easily follows from Proposition 1.1 that for each $n \in \mathbb{N} \mathcal{H}_{\tau, \mathbb{C}}^{\hat{\otimes} n}$ is densely and continuously embedded in $\mathcal{H}_{\mathrm{ext}}^{(n)}$, and estimate (1.5) with $\tau$ instead of 
$\tilde{\tau}$ ( $c$ depends on $\tau$ ) holds true. Therefore by analogy with Remarks $1.4,1.6$ one can exclude from $T$ indexes $\tau$ such that there is no a continuous embedding $\mathcal{H}_{\tau}$ in $\mathcal{H}_{\tilde{\tau}}$, and assume in what follows, that the results of Proposition 1.1 hold true for all $\tau$ $\in T$.

Now we can consider the chains

$$
D_{\mathbb{C}}^{\prime(n)} \supset \mathcal{H}_{-\tau, \mathbb{C}}^{(n)} \supset \mathcal{H}_{\mathrm{ext}}^{(n)} \supset \mathcal{H}_{\tau, \mathbb{C}}^{\hat{\otimes} n} \supset D_{\mathbb{C}}^{\hat{\otimes} n},
$$

where $\mathcal{H}_{-\tau, \mathbb{C}}^{(n)}, \quad D_{\mathbb{C}}^{\prime(n)}=$ ind $\lim _{\tau \in T} \mathcal{H}_{-\tau, \mathbb{C}}^{(n)}$ are the dual to $\mathcal{H}_{\tau, \mathbb{C}}^{\hat{\otimes} n}, \quad D_{\mathbb{C}}^{\hat{\otimes} n}$ with respect to $\mathcal{H}_{\mathrm{ext}}^{(n)}$ spaces correspondingly. For the generated by the scalar product in $\mathcal{H}_{\text {ext }}^{(n)}$ (real) dual pairings between elements of $D_{\mathbb{C}}^{\prime(n)}$ and $D_{\mathbb{C}}^{\hat{\otimes} n}$ (in the same way as $\mathcal{H}_{-\tau, \mathbb{C}}^{(n)}$ and $\left.\mathcal{H}_{\tau, \mathbb{C}}^{\hat{\otimes} n}\right)$ we preserve the notation $\langle\cdot, \cdot\rangle_{\text {ext }} \cdot$

Of course, for $n=1$ chain (1.6) has the form

$$
D_{\mathbb{C}}^{\prime} \supset \mathcal{H}_{-\tau, \mathbb{C}} \supset \mathcal{H}_{\mathrm{ext}}^{(1)}=\mathcal{H}_{\mathbb{C}} \supset \mathcal{H}_{\tau, \mathbb{C}} \supset D_{\mathbb{C}},
$$

i.e., this chain coincides with the complexification of chain (1.1). But for $n>1$ and $\eta \neq 0$ chain (1.6) is not a tensor power of chain of type (1.1). Nevertheless, there exists the natural interconnection between chains (1.4) and (1.6). In fact, since $D_{\mathbb{C}}^{\prime(n)}$ in the same way as $D_{\mathbb{C}}^{\prime \hat{\otimes} n}, \quad n \in \mathbb{Z}_{+}$, are the sets of linear continuous functionals on $D_{\mathbb{C}}^{\hat{\otimes} n}$, there exist linear bijective operators $U_{n}: D_{\mathbb{C}}^{\prime(n)} \rightarrow D_{\mathbb{C}}^{\prime \hat{\otimes} n}$ such that $\forall F_{\text {ext }}^{(n)} \in D_{\mathbb{C}}^{\prime(n)}$ $\forall f^{(n)} \in D_{\mathbb{C}}^{\hat{\otimes} n}$

$$
\left\langle U_{n} F_{\mathrm{ext}}^{(n)}, f^{(n)}\right\rangle=\left\langle F_{\mathrm{ext}}^{(n)}, f^{(n)}\right\rangle_{\mathrm{ext}} .
$$

By analogy, since for all $\tau \in T \mathcal{H}_{-\tau, \mathbb{C}}^{(n)}$ and $\mathcal{H}_{-\tau, \mathbb{C}}^{\hat{\otimes} n}$ are the sets of linear continuous functionals on $\mathcal{H}_{\tau, \mathbb{C}}^{\hat{\otimes} n}$, there exist linear isometrical bijective operators $U_{n, \tau}: \mathcal{H}_{-\tau, \mathbb{C}}^{(n)} \rightarrow \mathcal{H}_{-\tau, \mathbb{C}}^{\hat{\otimes} n}$ such that

$$
\forall F_{\text {ext }}^{(n)} \in \mathcal{H}_{-\tau, \mathbb{C}}^{(n)} \forall f^{(n)} \in \mathcal{H}_{\tau, \mathbb{C}}^{\hat{\otimes} n}:\left\langle U_{n, \tau} F_{\text {ext }}^{(n)}, f^{(n)}\right\rangle=\left\langle F_{\text {ext }}^{(n)}, f^{(n)}\right\rangle_{\text {ext }} .
$$

Proposition 1.2 [15]. For each $\tau \in T$ and each $n \in \mathbb{Z}_{+}$the restriction of the operator $U_{n}$ on $\mathcal{H}_{-\tau, \mathbb{C}}^{(n)}$ coincides with $U_{n, \tau}$.

Taking into acount Proposition 1.2, in what follows, we omit a subindex $\tau$ for operators $U_{n, \tau}$, i.e., we'll write always $U_{n}$ for such operators.

Remark 1.10. We note that for $n=0$ and $n=1$, in the same way as for $n \in \mathbb{Z}_{+}$ and $\eta=0 \quad U_{n}=i d$; but for $n>1$ and $\eta \neq 0 U_{n} \mathcal{H}_{\mathrm{ext}}^{(n)} \neq \mathcal{H}_{\mathbb{C}}^{\hat{\otimes} n}$. This fact was proved in [22] for $\eta \equiv 1$ (in the Gamma analysis), the proof in the general case can be constructed by analogy.

Let $\mathcal{P}$ be the set of all continuous polynomials on $D^{\prime}$. It follows from results of $[16,10]$ that any element of $\mathcal{P}$ can be presented in the form

$$
f=\sum_{n=0}^{N_{f}}\left\langle P_{n}, f^{(n)}\right\rangle, \quad f^{(n)} \in D_{\mathbb{C}}^{\hat{\otimes}_{n}}, \quad N_{f} \in \mathbb{Z}_{+} .
$$

We define on $\mathcal{P}$ a family of scalar products by setting for $f, g \in \mathcal{P}, \tau \in T, q \in \mathbb{N}$ 


$$
(f, g)_{\tau, q}:=\sum_{n=0}^{\min \left(N_{f}, N_{g}\right)}(n !)^{2} 2^{q n}\left(f^{(n)}, g^{(n)}\right)_{\tau},
$$

where $f^{(n)}, g^{(n)}$ are the kernels from decompositions (1.8) for $f$ and $g$ respectively. By $\|\cdot\|_{\tau, q}$ denote the corresponding norm, i.e., for $f \in \mathcal{P}$ of form (1.8) we have

$$
\|f\|_{\tau, q}^{2}=(f, \bar{f})_{\tau, q}=\sum_{n=0}^{N_{f}}(n !)^{2} 2^{q n}\left|f^{(n)}\right|_{\tau}^{2} .
$$

Definition 1.5. By $\left(\mathcal{H}_{\tau}\right)_{q}$ denote a Hilbert space that is the closure of $\mathcal{P}$ with respect to the norm $\|\cdot\|_{\tau, q}$. Let also

$$
\left(\mathcal{H}_{\tau}\right):=\operatorname{pr} \lim _{q \in \mathbb{N}}\left(\mathcal{H}_{\tau}\right)_{q}, \quad(D):=\operatorname{pr} \lim _{\tau \in T, q \in \mathbb{N}}\left(\mathcal{H}_{\tau}\right)_{q} .
$$

The spaces $\left(\mathcal{H}_{\tau}\right)_{q},\left(\mathcal{H}_{\tau}\right),(D)$ are called the Kondratiev-type test functions spaces.

It is clear that $f \in\left(\mathcal{H}_{\tau}\right)_{q}$ if and only if $f$ can be presented in the form

$$
f=\sum_{n=0}^{\infty}\left\langle P_{n}, f^{(n)}\right\rangle
$$

with $f^{(n)} \in \mathcal{H}_{\tau, \mathbb{C}}^{\hat{\otimes} n}$, and the series converges in the sense that

$$
\|f\|_{\tau, q}^{2}:=\|f\|_{\left(\mathcal{H}_{\tau}\right)_{q}}^{2}=\sum_{n=0}^{\infty}(n !)^{2} 2^{q n}\left|f^{(n)}\right|_{\tau}^{2}<\infty .
$$

Further, $f \in\left(\mathcal{H}_{\tau}\right)$ if and only if $f$ has form (1.9) and norm (1.10) is finite for all $q \in \mathbb{N}$; and $f \in(D)$ if and only if norm (1.10) for $f$ is finite for all $\tau \in T$ and $q \in \mathbb{N}$ (in this case, of course, the kernels from decomposition (1.9) $f^{(n)} \in D_{\mathbb{C}}^{\hat{\otimes} n}$ ).

Remark 1.11. Let $f, g \in\left(\mathcal{H}_{\tau}\right)_{q}$. Then

$$
(f, g)_{\left(\mathcal{H}_{\tau}\right)_{q}}=\sum_{n=0}^{\infty}(n !)^{2} 2^{q n}\left(f^{(n)}, g^{(n)}\right)_{\tau}
$$

where $f^{(n)}, g^{(n)} \in \mathcal{H}_{\tau, \mathbb{C}}^{\hat{\otimes} n}$ are the kernels from decompositions (1.9) for $f$ and $g$ respectively; therefore the system of the generalized Meixner polynomials plays a role of an orthogonal basis in $\left(\mathcal{H}_{\tau}\right)_{q}$.

In order to define the Kondratiev-type spaces of generalized functions we need the following proposition.

Proposition 1.3 [15]. There exists $q_{0} \in \mathbb{N}$ such that for all natural $q \geq q_{0}$ and for all $\tau \in T$ the dense of continuous embedding $\left(\mathcal{H}_{\tau}\right)_{q} \hookrightarrow\left(L^{2}\right)$ takes place (we remind that $T$ is modified in accordance with Remarks 1.4, 1.6, 1.9).

Remark 1.12. Let $\mathbb{N}_{q_{0}}:=\left\{q_{0}, q_{0}+1, \ldots\right\} \subseteq \mathbb{N}$. Then we can reformulate Proposition 1.3 as follows: for all $q \in \mathbb{N}_{q_{0}}$ and for all $\tau \in T$ the dense and continuous embedding $\left(\mathcal{H}_{\tau}\right)_{q} \hookrightarrow\left(L^{2}\right)$ takes place.

Now we can consider the chain

$$
\begin{gathered}
\left(D^{\prime}\right)^{\prime} \supset\left(\mathcal{H}_{-\tau}\right) \supset\left(\mathcal{H}_{-\tau}\right)_{-q} \supset\left(L^{2}\right) \supset\left(\mathcal{H}_{\tau}\right)_{q} \supset\left(\mathcal{H}_{\tau}\right) \supset(D), \\
q \in \mathbb{N}_{q_{0}}, \quad \tau \in T,
\end{gathered}
$$

where 


$$
\left(\mathcal{H}_{-\tau}\right)_{-q}, \quad\left(\mathcal{H}_{-\tau}\right)=\text { ind } \lim _{q \in \mathbb{N}_{q_{0}}}\left(\mathcal{H}_{-\tau}\right)_{-q},\left(D^{\prime}\right)^{\prime}=\text { ind } \lim _{q \in \mathbb{N}_{q_{0}}, \tau \in T}\left(\mathcal{H}_{-\tau}\right)_{-q}
$$

are the dual to $\left(\mathcal{H}_{\tau}\right)_{q},\left(\mathcal{H}_{\tau}\right),(D)$ with respect to $\left(L^{2}\right)$ spaces correspondingly.

Definition 1.6. The spaces $\left(\mathcal{H}_{-\tau}\right)_{-q}, \quad q \in \mathbb{N}_{q_{0}}, \quad \tau \in T, \quad\left(\mathcal{H}_{-\tau}\right),\left(D^{\prime}\right)^{\prime}$ are called the Kondratiev-type spaces of nonregular generalized functions.

Let us recall a construction of orthogonal bases in the spaces $\left(\mathcal{H}_{-\tau}\right)_{-q}$. For $F_{\text {ext }}^{(n)} \in$ $\in \mathcal{H}_{-\tau, \mathbb{C}}^{(n)}$ we define $\left\langle P_{n}, F_{\text {ext }}^{(n)}\right\rangle \in\left(\mathcal{H}_{-\tau}\right)_{-q}$ as the limit in $\left(\mathcal{H}_{-\tau}\right)_{-q}$ of a sequence of polynomials $\left\langle P_{n}, F_{k}^{(n)}\right\rangle$ such that $\mathcal{H}_{\tau, \mathbb{C}}^{\hat{\otimes} n} \ni F_{k}^{(n)} \rightarrow F_{\text {ext }}^{(n)}$ as $k \rightarrow \infty$ in $\mathcal{H}_{-\tau, \mathbb{C}}^{(n)}$ (the correctness of this definition was proved in [15]).

Theorem 1.3 [15]. A generalized function $F \in\left(\mathcal{H}_{-\tau}\right)_{-q}, \quad \tau \in T, q \in \mathbb{N}_{q_{0}}$, if and only if there exists a sequence

$$
\left(F_{\mathrm{ext}}^{(n)} \in \mathcal{H}_{-\tau, \mathbb{C}}^{(n)}\right)_{n=0}^{\infty}
$$

such that $F$ can be presented in the form

$$
F=\sum_{n=0}^{\infty}\left\langle P_{n}, F_{\mathrm{ext}}^{(n)}\right\rangle,
$$

where the series converges in $\left(\mathcal{H}_{-\tau}\right)_{-q}$, i.e., the norm

$$
\|F\|_{-\tau,-q}^{2}:=\|F\|_{\left(\mathcal{H}_{-\tau}\right)_{-q}}^{2}=\sum_{n=0}^{\infty} 2^{-q n}\left|F_{\text {ext }}^{(n)}\right|_{-\tau, \text { ext }}^{2}<\infty
$$

(here and below by $|\cdot|_{-\tau, \mathrm{ext}}$ denote the norms in $\left.\mathcal{H}_{-\tau, \mathbb{C}}^{(n)}\right)$. Furthermore, the system $\left\{\left\langle P_{n}, F_{\mathrm{ext}}^{(n)}\right\rangle: F_{\mathrm{ext}}^{(n)} \in \mathcal{H}_{-\tau, \mathbb{C}}^{(n)}, n \in \mathbb{Z}_{+}\right\}$plays a role of an orthogonal basis in $\left(\mathcal{H}_{-\tau}\right)_{-q}$ in the sense that for $F, G \in\left(\mathcal{H}_{-\tau}\right)_{-q}$

$$
(F, G)_{\left(\mathcal{H}_{-\tau}\right)_{-q}}=\sum_{n=0}^{\infty} 2^{-q n}\left(F_{\mathrm{ext}}^{(n)}, G_{\mathrm{ext}}^{(n)}\right)_{-\tau, \mathrm{ext}},
$$

where $F_{\mathrm{ext}}^{(n)}, G_{\mathrm{ext}}^{(n)} \in \mathcal{H}_{-\tau, \mathbb{C}}^{(n)}$ are the kernels from decompositions (1.12) for $F$ and $G$ correspondingly, $(\cdot, \cdot)_{-\tau, \text { ext }}$ is the scalar product in $\mathcal{H}_{-\tau, \mathbb{C}}^{(n)} \cdot$

Remark 1.13. It is easy to see that $F \in\left(\mathcal{H}_{-\tau}\right)$ (correspondingly $F \in\left(D^{\prime}\right)^{\prime}$ ) if and only if there exists sequence (1.11) such that $F$ can be presented in form (1.12) with finite norm (1.13) for some $q \in \mathbb{N}$ (correspondingly for some $q \in \mathbb{N}$ and some $\tau \in T)$.

Remark 1.14. Note that one can introduce the spaces $(D)_{q}:=\operatorname{pr} \lim _{\tau \in T}\left(\mathcal{H}_{\tau}\right)_{q}$, $q \in \mathbb{N}$, and the corresponding dual ones; but from "the point of view of this paper" these spaces are completely analogous to the spaces $\left(\mathcal{H}_{\tau}\right)_{q}$ and $\left(\mathcal{H}_{-\tau}\right)_{-q}$.

The generated by the scalar product in $\left(L^{2}\right)$ (real) dual pairing between elements of $\left(\mathcal{H}_{-\tau}\right)_{-q}$ and $\left(\mathcal{H}_{\tau}\right)_{q}$ (in the same way as $\left(\mathcal{H}_{-\tau}\right)$ and $\left(\mathcal{H}_{\tau}\right),\left(D^{\prime}\right)^{\prime}$ and $\left.(D)\right)$ will be denoted by $\langle\langle\cdot, \cdot\rangle\rangle$. It was shown in [15] that for a generalized function $F$ of form (1.12) and a test function $f$ of form (1.9)

$$
\langle\langle F, f\rangle\rangle=\sum_{n=0}^{\infty} n !\left\langle F_{\mathrm{ext}}^{(n)}, f^{(n)}\right\rangle_{\mathrm{ext}} .
$$

Now let us recall elements of the Wick calculus. 
Definition 1.7. For $F \in\left(D^{\prime}\right)^{\prime}$ we define an integral $S$-transform $(S F)(\lambda) \quad(\lambda$ belongs to some depending on $F$ neighborhood of zero in $D_{\mathbb{C}}$ ) by setting (see (1.2))

$$
(S F)(\lambda):=\langle\langle F,: \exp (\cdot ; \lambda):\rangle\rangle .
$$

The $S$-transform is well-defined because for each $F \in\left(D^{\prime}\right)^{\prime}$ there exist $\tau \in T$ and $q \in \mathbb{N}_{q_{0}}$ such that $F \in\left(\mathcal{H}_{-\tau}\right)_{-q} ;$ and for $\lambda \in D_{\mathbb{C}}$ such that $2^{q}|\lambda|_{\tau}^{2}<1$ we have $: \exp (\cdot ; \lambda): \in\left(\mathcal{H}_{\tau}\right)_{q}$.

Remark 1.15. It is easy to see that

$$
(S F)(\lambda)=\sum_{n=0}^{\infty}\left\langle F_{\mathrm{ext}}^{(n)}, \lambda^{\otimes n}\right\rangle_{\mathrm{ext}},
$$

where $F_{\mathrm{ext}}^{(n)} \in \mathcal{H}_{-\tau, \mathbb{C}}^{(n)}, \quad n \in \mathbb{N}_{+}$, are the kernels from decomposition (1.12) for $F$. In particular, $(S F)(0)=F_{\mathrm{ext}}^{(0)}, S 1 \equiv 1$.

Theorem 1.4 [16, 10]. The $S$-transform is a topological isomorphism between the space $\left(D^{\prime}\right)^{\prime}$ and the algebra $\mathrm{Hol}_{0}$ of germs of holomorphic at zero functions on $D_{\mathbb{C}}$.

Definition 1.8. For $F, G \in\left(D^{\prime}\right)^{\prime}$ and a holomorphic at $(S F)(0)$ function $h$ : $\mathbb{C} \rightarrow \mathbb{C}$ we define the Wick product $F \diamond G \in\left(D^{\prime}\right)^{\prime}$ and the Wick version of $h$ $h^{\diamond}(F) \in\left(D^{\prime}\right)^{\prime}$ by setting

$$
F \diamond G:=S^{-1}(S F \cdot S G), \quad h^{\diamond}(F):=S^{-1} h(S F) .
$$

The correctness of this definition from Theorem 1.4 follows.

Remark 1.16. It is easy to see that the Wick multiplication $\diamond$ is commutative, associative and distributive (over the field $\mathbb{C}$ ). Further, if $h$ from Definition 1.8 is presented in the form

$$
h(u)=\sum_{n=0}^{\infty} h_{n}(u-(S F)(0))^{n}
$$

then $h^{\diamond}(F)=\sum_{n=0}^{\infty} h_{n}(F-(S F)(0))^{\diamond n}$, where $F^{\diamond n}=\underbrace{F \diamond \ldots \diamond F}_{n \text { times }}$.

Let us write out the "coordinate form" of $F \diamond G$ and $h^{\diamond}(F)$ (this form is necessary for calculations). Let for $F_{\mathrm{ext}}^{(k)} \in D_{\mathbb{C}}^{\prime(k)}, G_{\mathrm{ext}}^{(m)} \in D_{\mathbb{C}}^{\prime(m)}$

$$
F_{\mathrm{ext}}^{(k)} \diamond G_{\mathrm{ext}}^{(m)}:=U_{k+m}^{-1}\left(U_{k} F_{\mathrm{ext}}^{(k)} \hat{\otimes} U_{m} G_{\mathrm{ext}}^{(m)}\right) \in D_{\mathbb{C}}^{\prime(k+m)}
$$

(see (1.7)). It is obvious that the "multiplication" $\diamond$ is commutative, associative and distributive (over the field $\mathbb{C}$ ). It was shown in [15] that

$$
\begin{gathered}
F \diamond G=\sum_{n=0}^{\infty}\left\langle P_{n}, \sum_{k=0}^{n} F_{\mathrm{ext}}^{(k)} \diamond G_{\mathrm{ext}}^{(n-k)}\right\rangle, \\
h^{\diamond}(F)=h_{0}+\sum_{n=1}^{\infty}\left\langle P_{n}, \sum_{k=1}^{n} h_{k} \sum_{m_{1}, \ldots, m_{k} \in \mathbb{N}: m_{1}+\ldots+m_{k}=n} F_{\mathrm{ext}}^{\left(m_{1}\right)} \diamond \ldots \diamond F_{\mathrm{ext}}^{\left(m_{k}\right)}\right\rangle,
\end{gathered}
$$

where $F_{\mathrm{ext}}^{(k)}, G_{\mathrm{ext}}^{(k)} \in D_{\mathbb{C}}^{\prime(k)}$ are the kernels from decompositions (1.12) for $F$ and $G$ correspondingly, $h_{k} \in \mathbb{C}, k \in \mathbb{Z}_{+}$, are the coefficients from decomposition (1.16) for $h$. 
Remark 1.17. It follows from (1.17) that, in particular,

$$
\left\langle P_{n}, F_{\mathrm{ext}}^{(n)}\right\rangle \diamond\left\langle P_{m}, G_{\mathrm{ext}}^{(m)}\right\rangle=\left\langle P_{n+m}, F_{\mathrm{ext}}^{(n)} \diamond G_{\mathrm{ext}}^{(m)}\right\rangle .
$$

This formula can be used in order to define the Wick product (and then the Wick version of a holomorphic function as a series) without the $S$-transform. Formulas (1.17) and (1.18) also can be used as definitions.

Let now $F, G \in\left(\mathcal{H}_{-\tau}\right), \quad \tau \in T$. Since the space $\left(\mathcal{H}_{-\tau}\right)$ is embedded in $\left(D^{\prime}\right)^{\prime}$, the Wick product $F \diamond G$ and the Wick version of a holomorphic at $(S F)(0)$ function $h h^{\diamond}$ are well-defined as elements of $\left(D^{\prime}\right)^{\prime}$ and "coordinate representations" (1.17), (1.18) hold true. Moreover, by Remark 1.13 and Proposition 1.2 the kernels from decompositions (1.17) and (1.18) are elements of $\mathcal{H}_{-\tau, \mathbb{C}}^{(n)}$

Theorem 1.5 [6]. Let $F, G \in\left(\mathcal{H}_{-\tau}\right), \tau \in T$, and $h: \mathbb{C} \rightarrow \mathbb{C}$ be a holomorphic at $(S F)(0)$ function. Then $F \diamond G \in\left(\mathcal{H}_{-\tau}\right)$ and $h^{\diamond}(F) \in\left(\mathcal{H}_{-\tau}\right)$. Moreover, the Wick multiplication is continuous in the topology of $\left(\mathcal{H}_{-\tau}\right)$.

Finally, we recall the definition of the extended stochastic integral in the Meixner analysis (see [15] for a detailed presentation).

Let $F \in\left(\mathcal{H}_{-\tau}\right) \otimes \mathcal{H}_{\mathbb{C}}$. It follows from Theorem 1.3 that $F$ can be presented in the form

$$
F(\cdot)=\sum_{n=0}^{\infty}\left\langle P_{n}, F_{\mathrm{ext},}^{(n)} \cdot\right\rangle, \quad F_{\mathrm{ext},}^{(n)}, \in \mathcal{H}_{-\tau, \mathbb{C}}^{(n)} \otimes \mathcal{H}_{\mathbb{C}}
$$

with

$$
\|F\|_{\left(\mathcal{H}_{-\tau}\right)_{-q}}^{2} \otimes \mathcal{H}_{\mathbb{C}}=\sum_{n=0}^{\infty} 2^{-q n}\left|F_{\text {ext, }}^{(n)} \cdot\right|_{\mathcal{H}_{-\tau, \mathbb{C}}^{(n)} \otimes \mathcal{H}_{\mathbb{C}}}^{2}<\infty
$$

for some $q \in \mathbb{N}$. For $t \in[0,+\infty]$ we set

$$
\hat{F}_{\text {ext, }[0, t)}^{(n)}:=U_{n+1}^{-1}\left[\operatorname{Pr}\left(U_{n}\left(F_{\text {ext, }, ~}^{(n)}\right) 1_{[0, t)}(\cdot)\right)\right] \in \mathcal{H}_{-\tau, \mathbb{C}}^{(n+1)},
$$

where $\operatorname{Pr}$ is the symmertization operator, $U_{n}, U_{n+1}$ are defined in (1.7), here and below $1_{A}$ denotes the indicator of a set $A$.

Let $\left\{M_{s}:=\left\langle P_{1}, 1_{[0, s)}\right\rangle\right\}_{s \geq 0}$ be the Meixner random process (this process is a locally square integrable normal martingale with independent instruments, see $[15,5]$ for more details).

Definition 1.9. Let $F \in\left(\mathcal{H}_{-\tau}\right) \otimes \mathcal{H}_{\mathbb{C}}, t \in[0,+\infty]$. We define an extended stochastic integral with respect to the Meixner process $\int_{0}^{t} F(s) \hat{d} M_{s} \in\left(\mathcal{H}_{-\tau}\right)$ by setting

$$
\int_{0}^{t} F(s) \hat{d} M_{s}:=\sum_{n=0}^{\infty}\left\langle P_{n+1}, \hat{F}_{\mathrm{ext},[0, t)}^{(n)}\right\rangle,
$$

where $\hat{F}_{\mathrm{ext},[0, t)}^{(n)} \in \mathcal{H}_{-\tau, \mathbb{C}}^{(n+1)}, \quad n \in \mathbb{Z}_{+}$, are constructed in (1.20) starting from the kernels $F_{\text {ext, }}^{(n)} \in \mathcal{H}_{-\tau, \mathbb{C}}^{(n)} \otimes \mathcal{H}_{\mathbb{C}}$ from decomposition (1.19) for $F$.

It was proved in [15] that this definition is correct and integral (1.21) is a generalization of the Itô stochastic integral with respect to $M$. The case $F \in\left(D^{\prime}\right)^{\prime} \otimes \mathcal{H}_{\mathbb{C}}$ is completely analogous to the case $F \in\left(\mathcal{H}_{-\tau}\right) \otimes \mathcal{H}_{\mathbb{C}}$ (see [15] for details).

The interconnection between the Wick calculus and the extended stochastic integration is given by the formula (see [15]) 


$$
\int_{0}^{t} F(s) \hat{d} M_{s}=\int_{0}^{t} F(s) \diamond M_{s}^{\prime} \sigma(d s),
$$

where $t \in[0,+\infty], \quad F \in\left(\mathcal{H}_{-\tau}\right) \otimes \mathcal{H}_{\mathbb{C}} \quad\left(\right.$ or $\left.\quad F \in\left(D^{\prime}\right)^{\prime} \otimes \mathcal{H}_{\mathbb{C}}\right), \quad\left\{M_{s}^{\prime}:=\left\langle P_{1}, \delta_{s}\right\rangle \in\right.$ $\left.\in\left(\mathcal{H}_{-\tau}\right)\right\}_{s \geq 0}$ (here $\delta$ is the Dirac delta-function) is the Meixner white noise (the mentioned in Theorem 1.1 generalized stochastic process).

2. Generalized stochastic derivatives. In this section we introduce and study generalized stochastic derivatives on the finite order spaces of generalized functions $\left(\mathcal{H}_{-\tau}\right)$ (here and below $\left.\tau \in T\right)$. As regards to the space $\left(D^{\prime}\right)^{\prime}$ one can easily repeat all our considerations by analogy. In the end of the section we consider a one example: calculate the generalized stochastic derivative of the solution of some stochastic equation with a Wick-type nonlinearity.

First let $\eta=0$ (this corresponds to the Gaussian and Poissonian cases). The generalization of the Hida stochastic derivative (see, e.g., [23]) $\partial$. can be defined now on $\left(\mathcal{H}_{-\tau}\right)$ as follows.

Definition 2.1. Let $F \in\left(\mathcal{H}_{-\tau}\right), \eta=0$. We define a generalized stochastic derivative $\partial . F \in\left(\mathcal{H}_{-\tau}\right) \otimes \mathcal{H}_{-\tau, \mathbb{C}}$ by setting

$$
\partial . F:=\sum_{n=1}^{\infty} n\left\langle P_{n-1}, F_{\mathrm{ext}}^{(n)}(\cdot)\right\rangle \equiv \sum_{n=0}^{\infty}(n+1)\left\langle P_{n}, F_{\mathrm{ext}}^{(n+1)}(\cdot)\right\rangle,
$$

where $F_{\mathrm{ext}}^{(n)}(\cdot) \in \mathcal{H}_{-\tau, \mathbb{C}}^{\hat{\otimes} n-1} \otimes \mathcal{H}_{-\tau, \mathbb{C}}, n \in \mathbb{N}$, are obtained from the kernels from decomposition (1.12) for $F$ by "separating of a one argument" (note that now $\left.\mathcal{H}_{-\tau, \mathbb{C}}^{(n)}=\mathcal{H}_{-\tau, \mathbb{C}}^{\hat{\otimes} n} \subset \mathcal{H}_{-\tau, \mathbb{C}}^{\hat{\otimes} n-1} \otimes \mathcal{H}_{-\tau, \mathbb{C}}\right)$.

For a general $\eta \neq 0$ this definition can not be accepted because for $n>1$ $\mathcal{H}_{-\tau, \mathbb{C}}^{(n)} \not \subset \mathcal{H}_{-\tau, \mathbb{C}}^{(n-1)} \otimes \mathcal{H}_{-\tau, \mathbb{C}}$, therefore the kernels $F_{\text {ext }}^{(n)} \in \mathcal{H}_{-\tau, \mathbb{C}}^{(n)}$ from (1.12) can not be considered as elements of $\mathcal{H}_{-\tau, \mathbb{C}}^{(n-1)} \otimes \mathcal{H}_{-\tau, \mathbb{C}}$. In order to "go around" this problem we accept the following definition.

Definition 2.2. For $F_{\mathrm{ext}}^{(n)} \in \mathcal{H}_{-\tau, \mathbb{C}}^{(n)}$ we define $F_{\mathrm{ext}}^{(n)}(\cdot) \in \mathcal{H}_{-\tau, \mathbb{C}}^{(n-1)} \otimes \mathcal{H}_{-\tau, \mathbb{C}}, n \in \mathbb{N}$, by the formula

$$
F_{\mathrm{ext}}^{(n)}(\cdot):=U_{n-1}^{-1}\left[\left(U_{n} F_{\mathrm{ext}}^{(n)}\right)(\cdot)\right],
$$

where the isomorphisms $U_{n}: \mathcal{H}_{-\tau, \mathbb{C}}^{(n)} \rightarrow \mathcal{H}_{-\tau, \mathbb{C}}^{\hat{\otimes} n}, n \in \mathbb{Z}_{+}$, are defined by (1.7).

Remark 2.1. Note that

$$
\left|F_{\mathrm{ext}}^{(n)}(\cdot)\right|_{\mathcal{H}_{-\tau, \mathbb{C}}^{(n-1)} \otimes \mathcal{H}_{-\tau, \mathbb{C}}}=\left|\left(U_{n} F_{\mathrm{ext}}^{(n)}\right)(\cdot)\right|_{\mathcal{H}_{-\tau, \mathbb{C}}^{\hat{\otimes}_{n} n-1} \otimes \mathcal{H}_{-\tau, \mathbb{C}}}=\left|U_{n} F_{\mathrm{ext}}^{(n)}\right|_{-\tau}=\left|F_{\mathrm{ext}}^{(n)}\right|_{-\tau, \mathrm{ext}} \cdot
$$

Remark 2.2. Since for $\eta=0 \quad U_{n}=i d$ for all $n \in \mathbb{Z}_{+}$, a defined by (2.2) $F_{\text {ext }}^{(n)}(\cdot)$ coincides in this case with $F_{\text {ext }}^{(n)}(\cdot)$ from Definition 2.1.

Now we are ready to give a definition of a generalized stochastic derivative on $\left(\mathcal{H}_{-\tau}\right)$.

Definition 2.3. Let $F \in\left(\mathcal{H}_{-\tau}\right)$. We define a generalized stochastic derivative $\partial . F \in\left(\mathcal{H}_{-\tau}\right) \otimes \mathcal{H}_{-\tau, \mathbb{C}}$ by formula $(2.1)$, where $F_{\mathrm{ext}}^{(n)}(\cdot) \in \mathcal{H}_{-\tau, \mathbb{C}}^{(n-1)} \otimes \mathcal{H}_{-\tau, \mathbb{C}}, \quad n \in \mathbb{N}$, 
are constructed in (2.2) starting from the kernels $F_{\mathrm{ext}}^{(n)} \in \mathcal{H}_{-\tau, \mathbb{C}}^{(n)}$ from decomposition (1.12) for $F$.

Remark 2.3. Not that for $\eta \neq 0$ the restriction of $\partial$. on $\left(L^{2}\right)$ does not coincide with the constructed in [15] "Hida derivative" on $\left(L^{2}\right)$. At the same time the "Hida derivative" on $\left(L^{2}\right)$ can not be continued on $\left(\mathcal{H}_{-\tau}\right)$. This is an objective fact that is connected with base properties of the Meixner measure.

Let us prove the correctness of Definition 2.3. Let $F \in\left(\mathcal{H}_{-\tau}\right)$. Then there exists $q \in \mathbb{N}$ such that $F \in\left(\mathcal{H}_{-\tau}\right)_{-q+1}$. Using this fact, (2.3), (2.1), and the simple estimate $\max _{n \in \mathbb{Z}_{+}}\left[(n+1)^{2} 2^{-n}\right]=9 / 4$, we obtain

$$
\begin{gathered}
\|\partial . F\|_{\left(\mathcal{H}_{-\tau}\right)_{-q} \otimes \mathcal{H}_{-\tau, \mathbb{C}}}^{2}=\sum_{n=0}^{\infty}(n+1)^{2} 2^{-q n}\left|F_{\mathrm{ext}}^{(n+1)}(\cdot)\right|_{\mathcal{H}_{-\tau, \mathbb{C}}^{(n)} \otimes \mathcal{H}_{-\tau, \mathbb{C}}}^{2}= \\
=\sum_{n=0}^{\infty}\left[(n+1)^{2} 2^{-n}\right] 2^{(-q+1) n}\left|F_{\mathrm{ext}}^{(n+1)}\right|_{-\tau, \mathrm{ext}}^{2} \leq \\
\leq 9 \cdot 2^{q-3} \sum_{n=0}^{\infty} 2^{(-q+1)(n+1)}\left|F_{\mathrm{ext}}^{(n+1)}\right|_{-\tau, \mathrm{ext}}^{2} \leq 9 \cdot 2^{q-3}|F|_{-\tau,-q+1}^{2}<\infty .
\end{gathered}
$$

Therefore $\partial$. is well-defined and, moreover, is a linear continuous operator acting from $\left(\mathcal{H}_{-\tau}\right)$ to $\left(\mathcal{H}_{-\tau}\right) \otimes \mathcal{H}_{-\tau, \mathbb{C}}$.

Let us calculate the adjoint to $\partial$. operator $\partial^{*}:\left(\mathcal{H}_{\tau}\right) \otimes \mathcal{H}_{\tau, \mathbb{C}} \rightarrow\left(\mathcal{H}_{\tau}\right)$. Let

$$
f(\cdot)=\sum_{m=0}^{\infty}\left\langle P_{m}, f^{(m)}\right\rangle \in\left(\mathcal{H}_{\tau}\right) \otimes \mathcal{H}_{\tau, \mathbb{C}}, \quad f^{(m)} \in \mathcal{H}_{\tau, \mathbb{C}}^{\hat{\otimes}_{m}} \otimes \mathcal{H}_{\tau, \mathbb{C}}
$$

(see (1.9)), $F \in\left(\mathcal{H}_{-\tau}\right)$. We have (see (1.12), (1.14), (1.7), (2.2))

$$
\begin{gathered}
(\partial . F, f(\cdot))_{\left(L^{2}\right) \otimes \mathcal{H}_{\mathbb{C}}}=\left(\sum_{n=0}^{\infty}(n+1)\left\langle P_{n}, F_{\mathrm{ext}}^{(n+1)}(\cdot)\right\rangle, \sum_{m=0}^{\infty}\left\langle P_{m}, f^{(m)}\right\rangle\right)_{\left(L^{2}\right) \otimes \mathcal{H}_{\mathbb{C}}}= \\
=\sum_{n=0}^{\infty}(n+1) !\left\langle F_{\mathrm{ext}}^{(n+1)}(\cdot), f^{(n)}\right\rangle_{\mathcal{H}_{\mathrm{ext}}^{(n)} \otimes \mathcal{H}_{\mathbb{C}}}= \\
=\sum_{n=0}^{\infty}(n+1) !\left\langle\left(U_{n+1} F_{\mathrm{ext}}^{(n+1)}\right)(\cdot), f^{(n)}\right\rangle_{\mathcal{H}_{\mathbb{C}}^{\otimes^{\otimes}} \otimes \mathcal{H}_{\mathbb{C}}}= \\
=\sum_{n=0}^{\infty}(n+1) !\left\langle U_{n+1} F_{\mathrm{ext}}^{(n+1)}, \operatorname{Pr} f^{(n)}\right\rangle=\sum_{n=0}^{\infty}(n+1) !\left\langle F_{\mathrm{ext}}^{(n+1)}, \operatorname{Pr} f^{(n)}\right\rangle_{\mathrm{ext}}= \\
=\left\langle\left\langle\sum_{n=0}^{\infty}\left\langle P_{n}, F_{\mathrm{ext}}^{(n)}\right\rangle, \sum_{m=0}^{\infty}\left\langle P_{m+1}, \operatorname{Pr} f^{(m)}\right\rangle\right\rangle\right\rangle=\left\langle\left\langle F, \partial^{*} f(\cdot)\right\rangle\right\rangle,
\end{gathered}
$$

where $\operatorname{Pr}$ is the symmetrization operator (more exactly, for $f^{(m)} \in \mathcal{H}_{\tau, \mathbb{C}}^{\hat{\otimes} m} \otimes$ $\otimes \mathcal{H}_{\tau, \mathbb{C}} \operatorname{Pr} f^{(m)} \in \mathcal{H}_{\tau, \mathbb{C}}^{\hat{\otimes}^{2} m+1}$ is a projection of $f^{(m)}$ on $\left.\mathcal{H}_{\tau, \mathbb{C}}^{\hat{\otimes} m+1}\right)$. Therefore, for $f(\cdot) \in\left(\mathcal{H}_{\tau}\right) \otimes \mathcal{H}_{\tau, \mathbb{C}}$ of form (2.4)

$$
\partial_{.}^{*} f(\cdot)=\sum_{m=0}^{\infty}\left\langle P_{m+1}, \operatorname{Pr} f^{(m)}\right\rangle .
$$


Remark 2.4. It is obvious that for $\eta=0$ and $f(\cdot) \in\left(\mathcal{H}_{\tau}\right) \otimes \mathcal{H}_{\tau, \mathbb{C}} \partial^{*} f(\cdot)=$ $=\int_{\mathbb{R}_{+}} f(s) \hat{d} M_{s}$. But for $\eta \neq 0 \int_{\mathbb{R}_{+}} f(s) \hat{d} M_{s} \notin\left(\mathcal{H}_{\tau}\right)$, generally speaking ( and, of course, $\partial^{*} \circ(\cdot)$ does not coincide with $\left.\int_{\mathbb{R}_{+}} \circ(s) \hat{d} M_{s}\right)$. Note also that even the integrability of $f(\cdot)$ by Itô is not sufficient for coincidence of $\partial^{*} f(\cdot)$ and $\int_{\mathbb{R}_{+}} f(s) \hat{d} M_{s}$ in the case $\eta \neq 0$.

Now let us obtain the analog of the Clack - Ocone theorem (see, e.g., [24 - 28]). We recall that the task consists in finding of the explicit expression for $m$, in the equality $F=\mathbf{E} F+\int_{\mathbb{R}_{+}} m_{s} \hat{d} M_{s} \quad$ (here and below $\mathbf{E}$ denotes the expectation: $\mathbf{E} F=$ $=\langle\langle F, 1\rangle\rangle=F^{(0)}=\left\langle P_{0}, F^{(0)}\right\rangle \in \mathbb{C}$, where $F^{(0)}$ is the kernel from decomposition (1.12) for $F$ ).

In the general case the following solution is possible. Let us introduce an operator $\partial^{\prime}, \in \mathcal{L}\left(\left(\mathcal{H}_{-\tau}\right)_{-q},\left(\mathcal{H}_{-\tau}\right)_{-q} \otimes \mathcal{H}_{-\tau, \mathbb{C}}\right) \quad\left(q \in \mathbb{N}_{q_{0}}, \mathcal{L}\left(H_{1}, H_{2}\right)\right.$ denotes the set of linear continuous operators acting from $H_{1}$ to $\left.H_{2}\right)$ by setting for $F \in\left(\mathcal{H}_{-\tau}\right)_{-q}$

$$
\partial^{\prime} F:=\sum_{n=1}^{\infty}\left\langle P_{n-1}, F_{\mathrm{ext}}^{(n)}(\cdot)\right\rangle,
$$

where $F_{\text {ext }}^{(n)}(\cdot) \in \mathcal{H}_{-\tau, \mathbb{C}}^{(n-1)} \otimes \mathcal{H}_{-\tau, \mathbb{C}}, n \in \mathbb{N}$, are constructed in (2.2) starting from the kernels $F_{\mathrm{ext}}^{(n)} \in \mathcal{H}_{-\tau, \mathbb{C}}^{(n)}$ from decomposition (1.12) for $F$. Since (see (2.3))

$$
\begin{gathered}
\left\|\partial^{\prime} . F\right\|_{\left(\mathcal{H}_{-\tau}\right)_{-q}}^{2} \otimes \mathcal{H}_{-\tau, \mathbb{C}}=\sum_{n=1}^{\infty} 2^{-q(n-1)}\left|F_{\mathrm{ext}}^{(n)}(\cdot)\right|_{\mathcal{H}_{-\tau, \mathbb{C}}^{(n-1)} \otimes \mathcal{H}_{-\tau, \mathbb{C}}}^{2}= \\
=2^{q} \sum_{n=1}^{\infty} 2^{-q n}\left|F_{\mathrm{ext}}^{(n)}\right|_{-\tau, \mathrm{ext}}^{2} \leq 2^{q}\|F\|_{-\tau,-q}^{2},
\end{gathered}
$$

this definition is correct.

Theorem 2.1 (cf. [29]). Let $F \in\left(\mathcal{H}_{-\tau}\right)$. Then

$$
F=\mathbf{E} F+\int_{\mathbb{R}_{+}} \partial_{s}^{\prime} F \hat{d} M_{s} .
$$

Proof. In fact, using (1.20) and (2.2) we obtain

$$
\int_{\mathbb{R}_{+}} \partial_{s}^{\prime} F \hat{d} M_{s}=\sum_{n=1}^{\infty}\left\langle P_{n}, U_{n}^{-1}\left(\operatorname{Pr}\left(U_{n-1} F_{\mathrm{ext}}^{(n)}(\cdot)\right)\right)\right\rangle=\sum_{n=1}^{\infty}\left\langle P_{n}, F_{\mathrm{ext}}^{(n)}\right\rangle .
$$

The theorem is proved.

Unfortunately, the using of the operator $\partial$., generally speaking, is impossible. But for a special particular case the following formal construction is possible (cf. [29]). Let $F \in\left(\mathcal{H}_{-\tau}\right)$ be such that $\forall n \in \mathbb{N}: U_{n} F_{\mathrm{ext}}^{(n)} \in \mathcal{H}_{\mathbb{C}}^{\hat{\otimes} n}$ (here $F_{\mathrm{ext}}^{(n)} \in \mathcal{H}_{-\tau, \mathbb{C}}^{(n)}$, $n \in \mathbb{Z}_{+}$, are the kernels from decomposition (1.12) for $F$ ). We define

$$
\mathbf{E}\left(\partial .\left.F\right|_{\mathcal{F}}\right):=\sum_{n=1}^{\infty} n\left\langle P_{n-1}, U_{n-1}^{-1}\left(\left(U_{n} F_{\mathrm{ext}}^{(n)}\right)(\cdot) 1_{[0,]^{n-1}}\right)\right\rangle
$$

(for $\eta=0$ and under some additional conditions the right-hand side of (2.6) is the conditional expectation of $\partial . F$ with respect to the generated by $M$ full $\sigma$-algebra $\mathcal{F}$. : $=\sigma\left(M_{u}: u \leq \cdot\right)$, cf. [29], therefore this notation is natural). Since 


$$
\begin{gathered}
\left|U_{n-1}^{-1}\left(\left(U_{n} F_{\mathrm{ext}}^{(n)}\right)(\cdot) 1_{[0,]^{n-1}}\right)\right|_{\mathcal{H}_{-\tau, \mathbb{C}}^{(n-1)} \otimes \mathcal{H}_{\mathbb{C}}}=\left|\left(U_{n} F_{\mathrm{ext}}^{(n)}\right)(\cdot) 1_{[0,]^{n-1}}\right|_{\mathcal{H}_{-\tau, \mathbb{C}}^{\otimes_{n-1} \otimes \mathcal{H}_{\mathbb{C}}}} \leq \\
\leq c(\tau)\left|\left(U_{n} F_{\mathrm{ext}}^{(n)}\right)(\cdot) 1_{[0,]^{n-1}}\right|_{\mathcal{H}_{\mathbb{C}}^{\hat{C}_{n-1}} \otimes \mathcal{H}_{\mathbb{C}}} \leq c(\tau)\left|\left(U_{n} F_{\mathrm{ext}}^{(n)}\right)(\cdot)\right|_{\mathcal{H}_{\mathbb{C}}^{\hat{\otimes}_{n-1}} \otimes \mathcal{H}_{\mathbb{C}}}= \\
=c(\tau)\left|\left(U_{n} F_{\mathrm{ext}}^{(n)}\right)\right|_{0}<\infty,
\end{gathered}
$$

each summand of series (2.6) is well-defined as an element of $\left(\mathcal{H}_{-\tau}\right) \otimes \mathcal{H}_{\mathbb{C}}$; but the series is formal in the sense that, generally speaking, it can diverge in $\left(\mathcal{H}_{-\tau}\right) \otimes \mathcal{H}_{\mathbb{C}}$. Integrating (2.6) term by term we obtain the formal equality

$$
\int_{\mathbb{R}_{+}} \mathbf{E}\left(\left.\partial_{s} F\right|_{\mathcal{F}_{s}}\right) \hat{d} M_{s}=\sum_{n=1}^{\infty} n\left\langle P_{n}, U_{n}^{-1}\left(\operatorname{Pr}\left(\left(U_{n} F_{\mathrm{ext}}^{(n)}\right)(\cdot) 1_{[0, \cdot]^{n-1}}\right)\right)\right\rangle
$$

Lemma 2.1. Let $F^{(n)} \in \mathcal{H}_{\mathbb{C}}^{\hat{\otimes} n}, n \in \mathbb{N}$. Then $\operatorname{Pr}\left(F^{(n)}(\cdot) 1_{[0,]^{n-1}}\right)=\frac{1}{n} F^{(n)}$ in $\mathcal{H}_{\mathbb{C}}^{\hat{\otimes}_{n}}$ (here $F^{(n)}(\cdot) \in \mathcal{H}_{\mathbb{C}}^{\hat{\otimes} n-1} \otimes \mathcal{H}_{\mathbb{C}}$ is obtained from $F^{(n)}$ by "separating of a one argument", $\operatorname{Pr}$ is the symmetrization operator).

Proof. Let $\dot{F}^{(n)} \in F^{(n)}$ be a representative of $F^{(n)} \quad\left(\dot{F}^{(n)}\right.$ is a function depending on $n$ variables). Without loss of generality we may take $\dot{F}^{(n)}$ to be a symmetric function (in fact, let $M$ be the set of processions $\left(t_{1}, \ldots, t_{n}\right)$ such that $\dot{F}^{(n)}\left(t_{1}, \ldots, t_{n}\right)$ is not symmetric, then $\left.\sigma^{\otimes n}(M)=0\right)$. We have

$$
\begin{gathered}
\operatorname{Pr}\left(\dot{F}^{(n)}\left(t_{1}, \ldots, t_{n}\right) 1_{\left[0, t_{n}\right]^{n-1}}\left(t_{1}, \ldots, t_{n-1}\right)\right)=\frac{1}{n}\left[\dot{F}^{(n)}\left(t_{1}, \ldots, t_{n}\right) 1_{\left[0, t_{n}\right]^{n-1}}\left(t_{1}, \ldots, t_{n-1}\right)+\right. \\
+\dot{F}^{(n)}\left(t_{n}, t_{1}, \ldots, t_{n-1}\right) 1_{\left[0, t_{n-1}\right]^{n-1}}\left(t_{n}, t_{1}, \ldots, t_{n-2}\right)+\ldots \\
\left.\ldots+\dot{F}^{(n)}\left(t_{2}, \ldots, t_{n}, t_{1}\right) 1_{\left[0, t_{1}\right]^{n-1}}\left(t_{2}, \ldots, t_{n}\right)\right] .
\end{gathered}
$$

If all $t_{j}, j \in\{1, \ldots, n\}$, are different, then only one term in the right-hand side is not equal to zero, hence by virtue of symmetry of $\dot{F}^{(n)}$

$$
\operatorname{Pr}\left(\dot{F}^{(n)}\left(t_{1}, \ldots, t_{n}\right) 1_{\left[0, t_{n}\right]^{n-1}}\left(t_{1}, \ldots, t_{n-1}\right)\right)=\frac{1}{n} \dot{F}^{(n)}\left(t_{1}, \ldots, t_{n}\right) .
$$

The processions with coinciding arguments can be ignored because the measure $\sigma^{\otimes n}$ of the set of such processions is equal to zero.

The lemma is proved.

Using the rezult of this lemma, we can rewrite (2.7) in the form

$$
\int_{\mathbb{R}_{+}} \mathbf{E}\left(\left.\partial_{s} F\right|_{\mathcal{F}_{s}}\right) \hat{d} M_{s}=\sum_{n=1}^{\infty}\left\langle P_{n}, F_{\mathrm{ext}}^{(n)}\right\rangle
$$

i.e.,

$$
F=\mathbf{E} F+\int_{\mathbb{R}_{+}} \mathbf{E}\left(\left.\partial_{s} F\right|_{\mathcal{F}_{s}}\right) \hat{d} M_{s}
$$

By analogy with $[1,2]$ we consider now another stochastic differential operator (this new operator is closely connected with $\partial$., see Proposition 2.1 below). We begin from some technical preparation.

For $F_{\mathrm{ext}}^{(m)} \in \mathcal{H}_{-\tau, \mathbb{C}}^{(m)}$ and $f^{(n)} \in \mathcal{H}_{\tau, \mathbb{C}}^{\hat{\otimes}_{n}}, m>n$, we define a "pairing" $\left(F_{\mathrm{ext}}^{(m)}, f^{(n)}\right)_{\mathrm{ext}} \in$ $\in \mathcal{H}_{-\tau, \mathbb{C}}^{(m-n)}$ by the equality 


$$
\left\langle\left(F_{\mathrm{ext}}^{(m)}, f^{(n)}\right)_{\mathrm{ext}}, g^{(m-n)}\right\rangle_{\mathrm{ext}}=\left\langle F_{\mathrm{ext}}^{(m)}, f^{(n)} \hat{\otimes} g^{(m-n)}\right\rangle_{\mathrm{ext}} \quad \forall g^{(m-n)} \in \mathcal{H} \underset{\tau, \mathbb{C}}{\hat{\otimes} m-n} .
$$

Since

$$
\begin{gathered}
\left|\left\langle F_{\text {ext }}^{(m)}, f^{(n)} \hat{\otimes} g^{(m-n)}\right\rangle_{\text {ext }}\right| \leq\left|F_{\text {ext }}^{(m)}\right|_{-\tau, \text { ext }}\left|f^{(n)} \hat{\otimes} g^{(m-n)}\right|_{\tau} \leq \\
\leq\left|F_{\text {ext }}^{(m)}\right|_{-\tau, \text { ext }}\left|f^{(n)}\right|_{\tau}\left|g^{(m-n)}\right|_{\tau},
\end{gathered}
$$

$\left(F_{\text {ext }}^{(m)}, f^{(n)}\right)_{\text {ext }}$ is well-defined as an element of $\mathcal{H}_{-\tau, \mathbb{C}}^{(m-n)}$ and, moreover,

$$
\left|\left(F_{\mathrm{ext}}^{(m)}, f^{(n)}\right)_{\mathrm{ext}}\right|_{-\tau, \mathrm{ext}} \leq\left|F_{\mathrm{ext}}^{(m)}\right|_{-\tau, \mathrm{ext}}\left|f^{(n)}\right|_{\tau} .
$$

For $m=n$ we set $\left(F_{\mathrm{ext}}^{(n)}, f^{(n)}\right)_{\mathrm{ext}}:=\left\langle F_{\mathrm{ext}}^{(n)}, f^{(n)}\right\rangle_{\mathrm{ext}}$.

Definition 2.4. For an arbitrary $f^{(n)} \in \mathcal{H}_{\tau, \mathbb{C}}^{\hat{\otimes} n}$ we define an operator $\left(\mathcal{D}^{n} \circ\right)\left(f^{(n)}\right) \in \mathcal{L}\left(\left(\mathcal{H}_{-\tau}\right),\left(\mathcal{H}_{-\tau}\right)\right)$ by setting for $F=\sum_{m=0}^{\infty}\left\langle P_{m}, F_{\mathrm{ext}}^{(m)}\right\rangle \in\left(\mathcal{H}_{-\tau}\right)$.

$$
\left(\mathcal{D}^{n} F\right)\left(f^{(n)}\right):=\sum_{m=0}^{\infty} \frac{(m+n) !}{m !}\left\langle P_{m},\left(F_{\mathrm{ext}}^{(m+n)}, f^{(n)}\right)_{\mathrm{ext}}\right\rangle \in\left(\mathcal{H}_{-\tau}\right) .
$$

Since for each $F \in\left(\mathcal{H}_{-\tau}\right)$ there exists $q \in \mathbb{N}$ such that $F \in\left(\mathcal{H}_{-\tau}\right)_{-q+2}$, we have

$$
\begin{gathered}
\left\|\left(\mathcal{D}^{n} F\right)\left(f^{(n)}\right)\right\|_{-\tau,-q}^{2}=\|\left.\sum_{m=0}^{\infty} \frac{(m+n) !}{m !}\left\langle P_{m},\left(F_{\mathrm{ext}}^{(m+n)}, f^{(n)}\right)_{\mathrm{ext}}\right\rangle\right|_{-\tau,-q} ^{2} \leq \\
\leq \sum_{m=0}^{\infty} 2^{-q m}\left(\frac{(m+n) !}{m !}\right)^{2}\left|F_{\mathrm{ext}}^{(m+n)}\right|_{-\tau, \mathrm{ext}}^{2}\left|f^{(n)}\right|_{\tau}^{2} \leq \\
\leq(n !)^{2}\left|f^{(n)}\right|_{\tau}^{2} \sum_{m=0}^{\infty} 2^{-q m} 2^{2(m+n)}\left|F_{\mathrm{ext}}^{(m+n)}\right|_{-\tau, \mathrm{ext}}^{2}= \\
=2^{q n}(n !)^{2}\left|f^{(n)}\right|_{\tau}^{2} \sum_{m=0}^{\infty} 2^{-(q-2)(m+n)}\left|F_{\mathrm{ext}}^{(m+n)}\right|_{-\tau, \mathrm{ext}}^{2} \leq \\
\leq 2^{q n}(n !)^{2}\left|f^{(n)}\right|_{\tau}^{2}\|F\|_{-\tau,-(q-2)}^{2}<\infty
\end{gathered}
$$

(we used the estimate $\frac{(m+n) !}{m !}=n ! C_{m+n}^{m} \leq n ! 2^{m+n}$ ). Therefore this definition is correct and, moreover, for each $F \in\left(\mathcal{H}_{-\tau}\right)\left(\mathcal{D}^{n} F\right)(\circ) \in \mathcal{L}\left(\mathcal{H}_{\tau, \mathbb{C}}^{\hat{\otimes} n},\left(\mathcal{H}_{-\tau}\right)\right)$.

Remark 2.5. Let $\mathcal{D}:=\mathcal{D}^{1}$. It is not difficult to show by the direct calculation that for $g_{1}^{(1)}, \ldots, g_{n}^{(1)} \in \mathcal{H}_{\tau, \mathbb{C}}$ and $F \in\left(\mathcal{H}_{-\tau}\right)$

$$
\left.\left.\left.(\underbrace{\mathcal{D}(\ldots(\mathcal{D}((\mathcal{D} F)}_{n \text { times }}\left(g_{1}^{(1)}\right))\right)\left(g_{2}^{(1)}\right) \ldots\right)\right)\left(g_{n}^{(1)}\right)=\left(\mathcal{D}^{n} F\right)\left(g_{1}^{(1)} \hat{\otimes} g_{2}^{(1)} \hat{\otimes} \ldots \hat{\otimes} g_{n}^{(1)}\right) \text {. }
$$

Theorem 2.2. For each $F \in\left(\mathcal{H}_{-\tau}\right)$ the kernels $F_{\mathrm{ext}}^{(n)} \in \mathcal{H}_{-\tau, \mathbb{C}}^{(n)}, \quad n \in \mathbb{Z}_{+}$, from decomposition (1.12) can be presented in the form

$$
F_{\mathrm{ext}}^{(n)}=\frac{1}{n !} \mathbf{E}\left(\mathcal{D}^{n} F\right)
$$

Proof. Using (2.9), for each $f^{(n)} \in \mathcal{H}_{\tau, \mathbb{C}}^{\hat{\otimes} n}$ we obtain 


$$
\mathbf{E}\left(\left(\mathcal{D}^{n} F\right)\left(f^{(n)}\right)\right)=\left\langle\left\langle\left(\mathcal{D}^{n} F\right)\left(f^{(n)}\right), 1\right\rangle\right\rangle=n !\left(F_{\mathrm{ext}}^{(n)}, f^{(n)}\right)_{\mathrm{ext}}=n !\left\langle F_{\mathrm{ext}}^{(n)}, f^{(n)}\right\rangle_{\mathrm{ext}},
$$

this equality can be formally rewritten in form (2.10).

The theorem is proved.

Let us calculate the adjoint to $\left(\mathcal{D}^{n} \circ\right)\left(f^{(n)}\right), \quad f^{(n)} \in \mathcal{H}_{\tau, \mathbb{C}}^{\hat{\otimes} n}$ operator. For $F \in\left(\mathcal{H}_{-\tau}\right)$ and $g \in\left(\mathcal{H}_{\tau}\right)$ we have (see (2.9), (1.9), (1.14), (2.8), (1.12))

$$
\begin{gathered}
\left\langle\left\langle\left(\mathcal{D}^{n} F\right)\left(f^{(n)}\right), g\right\rangle\right\rangle=\left\langle\left\langle\sum_{m=0}^{\infty} \frac{(m+n) !}{m !}\left\langle P_{m},\left(F_{\mathrm{ext}}^{(m+n)}, f^{(n)}\right)_{\mathrm{ext}}\right\rangle, \sum_{k=0}^{\infty}\left\langle P_{k}, g^{(k)}\right\rangle\right\rangle\right\rangle= \\
=\sum_{m=0}^{\infty}(m+n) !\left\langle\left(F_{\mathrm{ext}}^{(m+n)}, f^{(n)}\right)_{\mathrm{ext}}, g^{(m)}\right\rangle_{\mathrm{ext}}=\sum_{m=0}^{\infty}(m+n) !\left\langle F_{\mathrm{ext}}^{(m+n)}, f^{(n)} \hat{\otimes} g^{(m)}\right\rangle_{\mathrm{ext}}= \\
=\left\langle\left\langle\sum_{k=0}^{\infty}\left\langle P_{k}, F_{\mathrm{ext}}^{(k)}\right\rangle, \sum_{m=0}^{\infty}\left\langle P_{m+n}, f^{(n)} \hat{\otimes} g^{(m)}\right\rangle\right\rangle\right\rangle=\left\langle\left\langle F,\left(\mathcal{D}^{n} g\right)\left(f^{(n)}\right)^{*}\right\rangle\right\rangle,
\end{gathered}
$$

therefore

$$
\left(\mathcal{D}^{n} g\right)\left(f^{(n)}\right)^{*}=\sum_{m=0}^{\infty}\left\langle P_{m+n}, f^{(n)} \hat{\otimes} g^{(m)}\right\rangle,
$$

where $g^{(m)} \in \mathcal{H}_{\tau, \mathbb{C}}^{\hat{\otimes} m}, m \in \mathbb{Z}_{+}$, are the kernels from decomposition (1.9) for $g$.

Now we focus on the operator $\mathcal{D}=\mathcal{D}^{1}$. The interconnection between $\mathcal{D}$ and $\partial$. is given by the following proposition.

Proposition 2.1. For all $F \in\left(\mathcal{H}_{-\tau}\right), \quad f^{(1)} \in \mathcal{H}_{\tau, \mathbb{C}}$

$$
\left\langle\partial F, f^{(1)}(\cdot)\right\rangle=(\mathcal{D} F)\left(f^{(1)}\right) .
$$

Proof. For $F=\sum_{m=0}^{\infty}\left\langle P_{m}, F_{\text {ext }}^{(m)}\right\rangle \in\left(\mathcal{H}_{-\tau}\right), g=\sum_{n=0}^{\infty}\left\langle P_{n}, g^{(n)}\right\rangle \in\left(\mathcal{H}_{\tau}\right)$, $f^{(1)} \in \mathcal{H}_{\tau, \mathbb{C}}$ we have (see (2.1), (1.14), (2.2), (2.9))

$$
\begin{aligned}
& \left\langle\left\langle\left\langle\partial . F, f^{(1)}(\cdot)\right\rangle, g\right\rangle\right\rangle=\left\langle\langle\langle\partial . F, g\rangle\rangle, f^{(1)}(\cdot)\right\rangle= \\
& =\left\langle\left\langle\left\langle\sum_{m=0}^{\infty}(m+1)\left\langle P_{m}, F_{\mathrm{ext}}^{(m+1)}(\cdot)\right\rangle, \sum_{n=0}^{\infty}\left\langle P_{n}, g^{(n)}\right\rangle\right\rangle, f^{(1)}(\cdot)\right\rangle=\right. \\
& =\left\langle\sum_{n=0}^{\infty}(n+1) !\left\langle F_{\mathrm{ext}}^{(n+1)}(\cdot), g^{(n)}\right\rangle_{\mathrm{ext}}, f^{(1)}(\cdot)\right\rangle= \\
& =\left\langle\sum_{n=0}^{\infty}(n+1) !\left\langle\left(U_{n+1} F_{\mathrm{ext}}^{(n+1)}\right)(\cdot), g^{(n)}\right\rangle, f^{(1)}(\cdot)\right\rangle= \\
& =\sum_{n=0}^{\infty}(n+1) !\left\langle U_{n+1} F_{\mathrm{ext}}^{(n+1)}, g^{(n)} \hat{\otimes} f^{(1)}\right\rangle=\sum_{n=0}^{\infty}(n+1) !\left\langle F_{\mathrm{ext}}^{(n+1)}, g^{(n)} \hat{\otimes} f^{(1)}\right\rangle_{\mathrm{ext}}= \\
& =\sum_{n=0}^{\infty}(n+1) !\left\langle\left(F_{\mathrm{ext}}^{(n+1)}, f^{(1)}\right)_{\mathrm{ext}}, g^{(n)}\right\rangle_{\mathrm{ext}}= \\
& =\left\langle\left\langle\sum_{m=0}^{\infty}(m+1)\left\langle P_{m},\left(F_{\text {ext }}^{(m+1)}, f^{(1)}\right)_{\text {ext }}\right\rangle, \sum_{n=0}^{\infty}\left\langle P_{n}, g^{(n)}\right\rangle\right\rangle\right\rangle=\left\langle\left\langle(\mathcal{D} F)\left(f^{(1)}\right), g\right\rangle\right\rangle .
\end{aligned}
$$

Since $g \in\left(\mathcal{H}_{\tau}\right)$ is arbitrary, it follows from this calculation that (2.12) is true. 
Remark 2.6. Note that formally $\partial . \circ=(\mathcal{D} \circ)(\delta$. $)$, where $\delta$ is the Dirac deltafunction.

Taking into consideration the result of Proposition 2.1, we preserve for $\mathcal{D}$ the name "a generalized stochastic derivative", cf. $[1,2,3]$.

Remark 2.7. Comparing (2.5) and (2.11) (with $n=1$ ) one can conclude that for $f \cdot g^{(1)}(\cdot)=f \otimes g^{(1)} \in\left(\mathcal{H}_{\tau}\right) \otimes \mathcal{H}_{\tau, \mathbb{C}}$ (here $\left.f \in\left(\mathcal{H}_{\tau}\right), g^{(1)} \in \mathcal{H}_{\tau, \mathbb{C}}\right) \partial^{*} f \cdot g^{(1)}(\cdot)=$ $=(\mathcal{D} f)\left(g^{(1)}\right)^{*}$.

Now let us study an interconnection between the stochastic differentiation and the Wick calculus (cf. [1, 2]).

Theorem 2.3. The operator $\mathcal{D} \circ$ is a pre-image of the directional derivative of $S \circ$ under the $S$-transform, i.e., for all $F \in\left(\mathcal{H}_{-\tau}\right), g \in \mathcal{H}_{\tau, \mathbb{C}}$

$$
(\mathcal{D} F)(g)=S^{-1} D_{g}(S F),
$$

where $D_{g}$ denotes the directional derivative in the direction $g$. Formula (2.13) can be accepted as a definition of $\mathcal{D}$.

Proof. Let $F=\sum_{m=0}^{\infty}\left\langle P_{m}, F_{\text {ext }}^{(m)}\right\rangle \in\left(\mathcal{H}_{-\tau}\right), \quad g \in \mathcal{H}_{\tau, \mathbb{C}}$. We have (see (1.15) and Theorem 1.4)

$$
\begin{gathered}
(S F)(\lambda)=\sum_{m=0}^{\infty}\left\langle F_{\mathrm{ext}}^{(m)}, \lambda^{\otimes m}\right\rangle_{\mathrm{ext}} \in \mathrm{Hol}_{0}, \\
D_{g}(S F)(\lambda)=\sum_{m=1}^{\infty} m\left\langle F_{\mathrm{ext}}^{(m)}, \lambda^{\otimes m-1} \hat{\otimes} g\right\rangle_{\mathrm{ext}}= \\
=\sum_{m=0}^{\infty}(m+1)\left\langle\left(F_{\mathrm{ext}}^{(m+1)}, g\right)_{\mathrm{ext}}, \lambda^{\otimes m}\right\rangle_{\mathrm{ext}} \in \mathrm{Hol}_{0} .
\end{gathered}
$$

Applying to $D_{g}(S F)$ the inverse $S$-transform we obtain (see (2.9))

$$
\left(D^{\prime}\right)^{\prime} \ni S^{-1} D_{g}(S F)=\sum_{m=0}^{\infty}(m+1)\left\langle P_{m},\left(F_{\mathrm{ext}}^{(m+1)}, g\right)_{\mathrm{ext}}\right\rangle=(\mathcal{D} F)(g) \text {. }
$$

But since $(\mathcal{D} F)(g) \in\left(\mathcal{H}_{-\tau}\right) \subset\left(D^{\prime}\right)^{\prime}, \quad S^{-1} D_{g}(S F)$ is well-defined as an element of $\left(\mathcal{H}_{-\tau}\right)$. Hence $(2.13)$ is proved.

Corollary. The operator $\mathcal{D}$ is a differentiation with respect to the Wick product, i.e., for all $F, G \in\left(\mathcal{H}_{-\tau}\right)$ we have

$$
\mathcal{D}(F \diamond G)=(\mathcal{D} F) \diamond G+F \diamond(\mathcal{D} G) .
$$

Moreover, for each $n \in \mathbb{Z}_{+}, \quad F \in\left(\mathcal{H}_{-\tau}\right)$, and a holomorphic at $(S F)(0)$ function $h: \mathbb{C} \rightarrow \mathbb{C}$

$$
\begin{gathered}
\mathcal{D} F^{\diamond n}=n F^{\diamond n-1} \diamond(\mathcal{D} F), \\
\mathcal{D} h^{\diamond}(F)=h^{\prime \diamond}(F) \diamond(\mathcal{D} F),
\end{gathered}
$$

where $h^{\prime}$ is a usual derivative of $h$.

Proof. Using (2.13), for each $g \in \mathcal{H}_{\tau, \mathbb{C}}$ we obtain

$$
\begin{gathered}
(\mathcal{D}(F \diamond G))(g)=S^{-1} D_{g}(S(F \diamond G))=S^{-1} D_{g}(S F \cdot S G)= \\
=S^{-1}\left(\left(D_{g} S F\right) \cdot S G+S F \cdot\left(D_{g} S G\right)\right)=S^{-1}(S(\mathcal{D} F)(g) \cdot S G+S F \cdot S(\mathcal{D} G)(g))=
\end{gathered}
$$




$$
=(\mathcal{D} F)(g) \diamond G+F \diamond(\mathcal{D} G)(g) \text {, }
$$

i.e., (2.14) is proved. The first formula in (2.15) can be obtained from (2.14) by induction (the case $n=0$ is trivial), the second one is a consequence of the first one.

Finally, let us calculate a commutator between the extended stochastic integral and the generalized stochastic derivative (known as a fundamental theorem of the Malliavin calculus, cf. [30]).

Theorem 2.4. Let $F \in\left(\mathcal{H}_{-\tau}\right) \otimes \mathcal{H}_{\mathbb{C}}$. Then $\forall t \in[0,+\infty]$

$$
\left(\mathcal{D} \int_{0}^{t} F(s) \hat{d} M_{s}\right)(\circ)=\int_{0}^{t}(\mathcal{D} F(s))(\circ) \hat{d} M_{s}+\int_{0}^{t} F(s) \circ(s) \sigma(d s) .
$$

Proof. By definition,

$$
\int_{0}^{t} F(s) \hat{d} M_{s}=\sum_{n=0}^{\infty}\left\langle P_{n+1}, \hat{F}_{\mathrm{ext},[0, t)}^{(n)}\right\rangle,
$$

where $\hat{F}_{\text {ext, }[0, t)}^{(n)} \in \mathcal{H}_{-\tau, \mathbb{C}}^{(n+1)}, \quad n \in \mathbb{Z}_{+}$, are constructed in (1.20) starting from the kernels $F_{\text {ext, }}^{(n)} \in \mathcal{H}_{-\tau, \mathbb{C}}^{(n)} \otimes \mathcal{H}_{\mathbb{C}}$ from decomposition (1.19) for $F$. Further,

$$
\left(\mathcal{D} \int_{0}^{t} F(s) \hat{d} M_{s}\right)(\circ)=\sum_{n=0}^{\infty}(n+1)\left\langle P_{n},\left(\hat{F}_{\mathrm{ext},[0, t)}^{(n)}, \circ\right)_{\mathrm{ext}}\right\rangle .
$$

On the other hand,

$$
\begin{gathered}
(\mathcal{D} F(\cdot))(\circ)=\sum_{n=1}^{\infty} n\left\langle P_{n-1},\left(F_{\mathrm{ext},, \cdot}^{(n)}, \circ\right)_{\mathrm{ext}}\right\rangle, \\
\int_{0}^{t}(\mathcal{D} F(s))(\circ) \hat{d} M_{s}=\sum_{n=1}^{\infty} n\left\langle P_{n},\left(\widehat{F_{\mathrm{ext}, .}^{(n)}, \circ}\right)_{\mathrm{ext},[0, t)}\right\rangle, \\
\int_{0}^{t} F(s) \circ(s) \sigma(d s)=\sum_{n=0}^{\infty}\left\langle P_{n}, \int_{0}^{t} F_{\mathrm{ext}, s}^{(n)} \circ(s) \sigma(d s)\right\rangle .
\end{gathered}
$$

Therefore, it is sufficient to prove that for all $n \in \mathbb{Z}_{+}$

$$
(n+1)\left(\hat{F}_{\mathrm{ext},[0, t)}^{(n)}, \circ\right)_{\mathrm{ext}}=n\left(\widehat{F_{\mathrm{ext},}^{(n)}, \circ}\right)_{\mathrm{ext},[0, t)}+\int_{0}^{t} F_{\mathrm{ext}, s}^{(n)} \circ(s) \sigma(d s) .
$$

For $n=0$ this equality is obviously true. Let $n \in \mathbb{N}$. It is sufficient to verify that for all $f^{(n)} \in \mathcal{H}_{\tau, \mathbb{C}}^{\hat{\otimes} n}$ and for all $g \in \mathcal{H}_{\tau, \mathbb{C}}$

$$
\begin{gathered}
(n+1)\left\langle\left(\hat{F}_{\mathrm{ext},[0, t)}^{(n)}, g\right)_{\mathrm{ext}}, f^{(n)}\right\rangle_{\mathrm{ext}}=n\left\langle\left(\widehat{F_{\mathrm{ext},}^{(n)}, g}\right)_{\mathrm{ext},[0, t)}, f^{(n)}\right\rangle_{\mathrm{ext}}+ \\
+\left\langle\int_{0}^{t} F_{\mathrm{ext}, s}^{(n)} g(s) \sigma(d s), f^{(n)}\right\rangle_{\mathrm{ext}} .
\end{gathered}
$$

Using (1.20) we can rewrite the left-hand side of (2.17) as follows:

$$
\begin{gathered}
(n+1)\left\langle\left(\hat{F}_{\mathrm{ext},[0, t)}^{(n)}, g\right)_{\mathrm{ext}}, f^{(n)}\right\rangle_{\mathrm{ext}}=(n+1)\left\langle\hat{F}_{\mathrm{ext},[0, t)}^{(n)}, g \hat{\otimes} f^{(n)}\right\rangle_{\mathrm{ext}}= \\
=(n+1)\left\langle\operatorname{Pr}\left(U_{n} F_{\mathrm{ext}, \cdot}^{(n)} 1_{[0, t)}(\cdot)\right), g \hat{\otimes} f^{(n)}\right\rangle=(n+1)\left\langle U_{n} F_{\mathrm{ext}, .}^{(n)} \cdot 1_{[0, t)}(\cdot), g \hat{\otimes} f^{(n)}\right\rangle= \\
=(n+1) \int_{0}^{t}\left\langle U_{n} F_{\mathrm{ext}, s}^{(n)},\left(g \hat{\otimes} f^{(n)}\right)(s)\right\rangle \sigma(d s)=\int_{0}^{t}\left\langle U_{n} F_{\mathrm{ext}, s}^{(n)}, f^{(n)}\left(\cdot{ }_{1}, \ldots,{ }_{n}\right) g(s)+\right.
\end{gathered}
$$




$$
\begin{gathered}
\left.+f^{(n)}\left(\cdot{ }_{2}, \ldots, s\right) g\left(\cdot \cdot_{1}\right)+\ldots+f^{(n)}\left(s, \ldots,{ }_{n-1}\right) g\left(\cdot{ }_{n}\right)\right\rangle \sigma(d s)= \\
=\int_{0}^{t}\left\langle U_{n} F_{\mathrm{ext}, s}^{(n)}, f^{(n)}\right\rangle g(s) \sigma(d s)+ \\
+\int_{0}^{t}\left\langle U_{n} F_{\mathrm{ext}, s}^{(n)}, f^{(n)}\left(\cdot{ }_{2}, \ldots, s\right) g\left(\cdot{ }_{1}\right)+\ldots+f^{(n)}\left(s, \ldots,{ }_{n-1}\right) g\left(\cdot{ }_{n}\right)\right\rangle \sigma(d s) .
\end{gathered}
$$

In the right-hand side of (2.17) we have

$$
\begin{gathered}
\left\langle\int_{0}^{t} F_{\mathrm{ext}, s}^{(n)} g(s) \sigma(d s), f^{(n)}\right\rangle_{\mathrm{ext}}=\int_{0}^{t}\left\langle F_{\mathrm{ext}, s}^{(n)}, f^{(n)}\right\rangle_{\mathrm{ext}} g(s) \sigma(d s)= \\
=\int_{0}^{t}\left\langle U_{n} F_{\mathrm{ext}, s}^{(n)}, f^{(n)}\right\rangle g(s) \sigma(d s)
\end{gathered}
$$

and by virtue of the symmetry of $f^{(n)}$ and (1.20)

$$
\begin{gathered}
n\left\langle\left(\widehat{F_{\mathrm{ext},}^{(n)}, g}\right)_{\mathrm{ext},[0, t)}, f^{(n)}\right\rangle_{\mathrm{ext}}=n\left\langle\operatorname{Pr}\left(U_{n-1}\left(F_{\mathrm{ext}, .}^{(n)}, g\right)_{\mathrm{ext}} 1_{[0, t)}(\cdot)\right), f^{(n)}\right\rangle= \\
=n\left\langle U_{n-1}\left(F_{\mathrm{ext}, .}^{(n)}, g\right)_{\mathrm{ext}} 1_{[0, t)}(\cdot), f^{(n)}\right\rangle=n \int_{0}^{t}\left\langle U_{n-1}\left(F_{\mathrm{ext}, s}^{(n)}, g\right)_{\mathrm{ext}}, f^{(n)}(s)\right\rangle \sigma(d s)= \\
=n \int_{0}^{t}\left\langle\left(F_{\mathrm{ext}, s}^{(n)}, g\right)_{\mathrm{ext}}, f^{(n)}(s)\right\rangle_{\mathrm{ext}} \sigma(d s)=n \int_{0}^{t}\left\langle F_{\mathrm{ext}, s}^{(n)}, g \hat{\otimes} f^{(n)}(s)\right\rangle_{\mathrm{ext}} \sigma(d s)= \\
=n \int_{0}^{t}\left\langle U_{n} F_{\mathrm{ext}, s}^{(n)}, g \hat{\otimes} f^{(n)}(s)\right\rangle \sigma(d s)=\int_{0}^{t}\left\langle U_{n} F_{\mathrm{ext}, s}^{(n)}, f^{(n)}\left(\cdot{ }_{2}, \ldots,{ }_{n}, s\right) g\left(\cdot{ }_{1}\right)+\right. \\
\left.\quad+f^{(n)}\left(\cdot_{3}, \ldots, s, \cdot_{1}\right) g\left(\cdot{ }_{2}\right)+\ldots+f^{(n)}\left(s,{ }_{1}, \ldots,{ }_{n-1}\right) g\left(\cdot{ }_{n}\right)\right\rangle \sigma(d s),
\end{gathered}
$$

thus (2.17) is proved.

By analogy with $[1,2]$ as an application of our results we will calculate the generalized stochastic derivative of the solution of the stochastic equation

$$
\left(\mathcal{H}_{-\tau}\right) \ni F_{t}=F_{0}+\int_{0}^{t} h^{\diamond}\left(F_{s}\right) \hat{d} M_{s},
$$

where $h: \mathbb{C} \rightarrow \mathbb{C}$ is some entire function, $F_{0} \in \mathbb{C}$. Under certain conditions on $h$ a unique solution of (2.18) $\quad F_{t} \in\left(\mathcal{H}_{-\tau}\right)$ exists. Applying $\mathcal{D}$ to (2.18) and taking into account (2.16) and (2.15), for each $g \in \mathcal{H}_{\tau, \mathbb{C}}$ we obtain

$$
\begin{gathered}
\left(\mathcal{D} F_{t}\right)(g)=\left(\mathcal{D} \int_{0}^{t} h^{\diamond}\left(F_{s}\right) \hat{d} M_{s}\right)(g)= \\
=\int_{0}^{t} h^{\prime \diamond}\left(F_{s}\right) \diamond\left(\mathcal{D} F_{s}\right)(g) \hat{d} M_{s}+\int_{0}^{t} h^{\diamond}\left(F_{s}\right) g(s) \sigma(d s) .
\end{gathered}
$$

Let $\phi_{S}^{g}(\lambda):=S\left(\left(\mathcal{D} F_{S}\right)(g)\right)(\lambda)$. Applying the $S$-transform to (2.19) and taking into account (1.22) we obtain

$$
\phi_{t}^{g}(\lambda)=\int_{0}^{t} h^{\prime}\left(\left(S F_{s}\right)(\lambda)\right) \phi_{s}^{g}(\lambda) \lambda(s) \sigma(d s)+\int_{0}^{t} h\left(\left(S F_{s}\right)(\lambda)\right) g(s) \sigma(d s) .
$$


The solution of this equation is

$$
\phi_{t}^{g}(\lambda)=\int_{0}^{t} h\left(\left(S F_{s}\right)(\lambda)\right) g(s) \cdot \exp \left\{\int_{s}^{t} h^{\prime}\left(\left(S F_{u}\right)(\lambda)\right) \lambda(u) \sigma(d u)\right\} \sigma(d s) .
$$

By the inverse $S$-transform we obtain

$$
\left(\mathcal{D} F_{t}\right)(g)=\int_{0}^{t} h^{\diamond}\left(F_{s}\right) g(s) \diamond \exp ^{\diamond}\left\{\int_{s}^{t} h^{\prime \diamond}\left(\left(F_{u}\right) \hat{d} M_{u}\right\} \sigma(d s) \in\left(\mathcal{H}_{-\tau}\right) .\right.
$$

Remark 2.8. It is not difficult to understand that main results of this paper can be reformulated "on the language of a so-called $Q$-system" under the biorthogonal approach to construction of a non-Gaussian infinite-dimensional analysis (see, e.g., [3, 6 $10,17,18]$ and references therein) and, therefore, can be applied in a more general case than the Meixner analysis. This follows from the fact that the $Q$-system is an orthogonal basis in the spaces $\left(\mathcal{H}_{-\tau}\right)_{-q}$ and $Q_{n}\left(F^{(n)}\right)=\left\langle P_{n}, U_{n}^{-1} F^{(n)}\right\rangle$, see [15].

1. Benth F. E. The Gross derivative of generalized random variables // Infin. Dimens. Anal. Quant. Probab. and Relat. Top. - 1999. - 2, № 3. - P. $381-396$.

2. Kachanovsky N.A. A generalized Malliavin derivative connected with the Poisson- and Gammameasures // Meth. Funct. Anal. and Top. - 2003. - 9, № 3. - P. 213 - 240.

3. Kachanovsky N. A. A generalized stochastic derivative on the Kondratiev-type spaces of regular generalized functions of Gamma white noise // Ibid. - 2006. - 12, № 4. - P. 363 - 383.

4. Kondratiev Yu. G., Luis da Silva J., Streit L., Us G. F. Analysis on Poisson and Gamma spaces // Infin. Dimens. Anal., Quant. Probab. and Relat. Top. - 1998. - 1, № 1. - P. 91 - 117.

5. Rodionova I. V. Analysis connected with generalized functions of exponential type in one and infinite dimensions // Meth. Funct. Anal. and Top. - 2005. - 11, № 3. - P. 275 - 297.

6. Kachanovsky N. A. Dual Appell-like systems and finite order spaces in non-Gaussian infinitedimensional analysis // Ibid. - 1998. - 4, № 2. - P. $41-52$.

7. Albeverio S., Kondratiev $Y u$. G., Streit $L$. How to generalize white noise analysis to non-Gaussian spaces // Dynam. Complex and Irregular Syst. / Eds Ph. Blanchard et. al. - World Sci., 1993. $20 \mathrm{p}$.

8. Albeverio S., Daletsky Yu. L., Kondratiev Yu. G., Streit L. Non-Gaussian infinite-dimensional analysis // J. Funct. Anal. - 1996. - 138, № 2. - P. 311 - 350.

9. Kondratiev Yu. G., Streit L., Westerkamp W., Yan J. Generalized functions in infinite-dimensional analysis // Hiroshima Math. J. - 1998. - 28. - P. 213 - 260.

10. Kondratiev Yu. G., Luis da Silva J., Streit L. Generalized Appell systems // Meth. Funct. Anal. and Top. - 1997. - 3, № 3. - P. $28-61$.

11. Berezansky Yu. M., Sheftel Z. G., Us G. F. Functional analysis. Vol. II // Operator Theory: Adv. and Appl. - Basel: Birkhäuser, 1996. - 86. - 312 p.

12. Gelfand I. M., Vilenkin N. Ya. Generalized functions. - New York; London: Acad. Press, 1964. Vol. 4. $-472 \mathrm{p}$.

13. Meixner J. Orthogonale Polynomsysteme mit einer besonderen Gestalt der erzeugenden Funktion // J. London Math. Soc. - 1934. - 9, № 1. - P. 6 - 13.

14. Litvinov E. W. Polynomials of Meixner's type in infinite dimensions Jacobi fields and orthogonality measures // J. Funct. Anal. - 2003. - 200. - P. $118-149$.

15. Kachanovsky N.A. On an extended stochastic integral and the Wick calculus on the connected with the generalized Meixner measure Kondratiev-type spaces // Meth. Funct. Anal. and Top. - 2007. 13, № 4. - P. $338-379$.

16. Kachanovsky N. A. Biorthogonal Appell-like systems in a Hilbert space // Ibid. - 1996. - 2, № 3. P. $33-52$.

17. Berezansky $Ү$ и.. . Infinite-dimensional analysis, related to generalized translation operator // Ukr. Math. J. - 1997. - 49, № 3. - P. $403-450$.

18. Berezansky Yu. M., Tesko V. A. Spaces of fundamental and generalized functions associated with generalized translation // Ibid. - 2003. - 55, № 12. - P. $1587-1658$.

19. Dineen $S$. Complex analysis in locally convex spaces // Math. Stud. - 1981. -57. -215 p.

20. Berezansky Yu. M., Merzejewski D. A. The structure of extended symmetric Fock space // Meth. Funct. Anal. and Top. - 2000. - 6, № 4. - P. $1-13$.

21. Grothaus M., Kondratiev Yu. G., Streit L. Regular generalized functions in Gaussian analysis infinite // Dimens. Anal. Quant. Probab. and Relat. Top. - 1999. - 2, № 3. - P. 359 - 380.

22. Kachanovsky N.A. On the extended stochastic integral connected with the Gamma-measure on an 
infinite-dimensional space // Meth. Funct. Anal. and Top. - 2002. - 8, № 2. - P. 10 - 32.

23. Hida T., Кио H. H., Potthoff J., Streit L. White noise // Infinite Dimensional Calculus. Dordrecht: Kluwer, 1993. - XIII+516 p.

24. Clark J. M. The representation of functionals of Brownian motion by stochastic integrals // Ann. Math. Statist. - 1970. - 41. - P. $1282-1291$.

25. Ocone D. Malliavin's calculus and stochastic integral: representation of functionals of diffusion processes // Stochastics. - 1984. - 12. - P. 161 - 185.

26. Lokka A. Martingale representation, chaos expansion and Clark - Ocone formulas // Res. Rept. Centre Math. Phys. and Stochast. Univ. Aarhus, Denmark. - 1999. - 22. - 24 p.

27. Aase K., Oksendal B., Privault N., Uboe J. White noise generalizations of the Clark - Haussmann Ocone theorem with application to mathematical finance // Finance Stochast. - 2000. - 4. P. $465-496$.

28. Di Nunno G., Oksendal B., Proske F. White noise analysis for Lévy processes // J. Funct. Anal. 2004. - 206. - P. 109 - 148.

29. Kachanovsky N. A. A generalized Clark - Ocone formula in non-Gaussian analysis // Spectral and Evolution. Problems. - 2000. - 10. - P. 66 - 71.

30. Di Nunno G., Meyer-Brandis T., Oksendal B., Proske F. Malliavin calculus and anticipative Itô formulae for Lévy processes // Infin. Dimens. Anal. Quant. Probab. and Relat. Top. - 2005. - 8, № 2. - P. $235-258$.

Received 28.12.06 